\title{
Tightly Coupled Navigation and Wind Estimation for Mini UAVs
}

\author{
Martin Brossard, Jean-Philippe Condomines ${ }^{\dagger}$ and Silvère Bonnabel* \\ ${ }^{*}$ Centre for Robotics, MINES ParisTech, 60 Boulevard Saint-Michel, 75006, Paris, France \\ †École Nationale de l'Aviation Civile, 7 Avenue Édouard Belin, 31055 Toulouse Cedex 4, France
}

\begin{abstract}
A novel nonlinear filter for the state-space model based on aided Inertial Navigation System, 1D Pitot static tube, angle-of-attack and angle-of-sideslip sensors is proposed. The solution, based on Multiplicative Kalman filtering, estimates in real-time the orientation, the velocity and the position of an Unmanned Aerial Vehicle along with sensor bias and 3D wind components. This paper describes and justifies the designed tightly coupled estimation scheme with both theoretical and experimental considerations. We validate then the whole approach for a mini UAV controlled through the well-known Paparazzi autopilot system which we equip with a set of low-cost sensors (accelerometers, gyros, GPS, magnetometer, barometer, 1D Pitot static tube and angular sensors), by successfully comparing the estimates obtained from real flight data with the $3 \mathrm{D}$ wind ground truth provided from a $60-\mathrm{m}$ weather measurement tower.
\end{abstract}

\section{Nomenclature}

z state vector

q unit quaternion

$\mathbf{R}_{\mathbf{q}}$ direction cosine matrix parametrized by quaternion $\mathbf{q}$

$\mathbf{v}$ UAV velocity in inertial frame

$\mathbf{x}$ UAV position in inertial frame

$\boldsymbol{\omega}_{b} \quad$ angular rate bias

$\mathbf{a}_{b} \quad$ acceleration bias in body frame

w wind velocity in inertial frame

$\mathbf{v}_{a} \quad$ airspeed in body frame

$\boldsymbol{\omega}$ biased angular velocity from gyro

a biased acceleration in body frame from accelerometer

$\alpha \quad$ Angle-of-Attack (AoA)

$\beta \quad$ Angle-of-Sideslip (AoS)

g gravity field vector

b magnetic field vector

Q process noise covariance matrix

$\mathbf{N}$ measurement noise covariance matrix

\section{Introduction}

The knowledge of the instantaneous wind velocity constitutes a precious information for Unmanned Aircraft Vehicles (UAVs), e.g., for atmospheric energy harvesting, wind disturbance rejection, target tracking, ${ }^{1-5}$ since wind significantly impacts flight performances, particularly for small and light vehicles. RIt may be useful to utilize fleets of UAVs to map the wind, e.g., for meteorological purposes, gas mapping, ${ }^{6-9}$ with several advantages over manned flight as low cost or simplicity of deployment. However, such mapping procedures usually assume the local wind estimation, i.e., the real-time estimation at the current location of the UAV, as an output of a yet established black-box, whereas it strongly affects the mapping quality. 
To estimate the local wind onboard an aircraft, one method consists in comparing the aircraft dynamical model and the wind velocity. ${ }^{10}$ Such techniques require accurately knowing the aircraft aerodynamic which is a clear limitation for mini UAVs, especially when the assumed steady state conditions are violated. For these reasons, we focus on the other main approach based on the wind triangle relationship which relates the airspeed, the ground speed and the wind speed. ${ }^{11-13}$ With this approach, Langelaan et al. investigate direct computations of the wind through simulation. ${ }^{13}$ Van den Kroonenberg et al. ${ }^{14}$ assume the horizontal wind speed as constant and no vertical wind during one flight loop and proposes then an experimental estimator through Kalman filtering. Similar estimators with no "flow sensor" have been tested, ${ }^{8,9}$ but an accurate 3D wind estimation need additional sensors, ${ }^{15}$ e.g., Pitot tube, pressure strip or vanes. ${ }^{7}$ With an added Pitot tube, Cho et al. ${ }^{16}$ design a relatively simple nonlinear Kalman filter for horizontal wind estimation. Recent works ${ }^{17-19}$ expand upon this concept to include vertical wind, incorporate Angle-ofAttack (AoA) and Angle-of-Sideslip (AoS) which both relate the aircraft body frame to the wind frame, and then uses Kalman filters to estimate attitude, ground speed and wind components. Such techniques require the capability to accurately determining attitude and speeds from various cheap sensors such as Global Positioning System (GPS), Inertial Measurement Unit (IMU) and magnetometer which is related to the UAV navigation problem, a well developed field ${ }^{20-24}$ in which, however, most existing works implicitly assume that the aircraft is always pointing in the direction of its total velocity, i.e., its AoA and AoS are negligible. Finally, validating such wind estimators in experimental flight is challenging due to the difficulty in accurately measuring the wind. One can yet use ground weather station data, radiosondes and piloted balloons, or SOnic Detection and Ranging, ${ }^{8,14-17}$ albeit only an approximation to the local wind at the aircraft.

Our approach assumes the low-cost sensor suite for navigation, i.e., GPS, IMU, magnometer and barometer, with additional 1D Pitot static tube and angle vanes. we mounted on our experimental drone. We opt for a sensor fusion scheme where navigation and wind estimation are tightly coupled. The contributions of the present paper are as follows

- As explained in the sequel, there are possible choices for the state variables. The one proposed here has not been much used yet for tightly coupled navigation and wind estimation.

- The fusion is performed by an (quaternion based Multiplicative) extended Kalman filter (M-EKF). Part of the initial nonlinear problem is linearized resorting to the virtual measurements technique, ${ }^{25,26}$ to handle our specific AoA and AoS measurements.

- The wind model is based on experimentally collected measurements of the wind.

- Monte-Carlo simulations using the software Paparazzi illustrate the good performances of the filter, and shows it competes with wind estimators of previous literature.

- We present validation flight experimental results, where a $60-\mathrm{m}$ measurement tower provides 3D wind "ground truth".

The rest of the paper is organized as follows. Section II defines and describes the considered model followed by the different approaches to perform filtering. In Section III, we design a MEKF-Wind filter for tightly coupled navigation and wind estimation. In Section IV parameter tuning is discussed, and experimentally obtained wind statistics support the chosen wind stochastic model and allow determining its noise amplitude. Section V illustrates the filter performances on realistic simulations. Section VI presents then experimental results, compared to data provided from a 60-m measurement tower, and we finally draw our conclusions in Section VII. Lower case non bold, lower case bold and upper case bold letters refer to, respectively, scalars, vectors or quaternions, and matrices.

\section{Quantities of Interest, Measurements, and Estimation Schemes}

This section presents the considered estimation problem, describes the various measurements available on our UAV, and then discusses various estimation schemes. 


\section{A. Dynamical Model}

Taking into account the wind velocity along with UAV navigation states, we define the dynamic model as $^{12,17}$

$$
\begin{aligned}
\dot{\mathbf{q}} & =\frac{1}{2} \mathbf{q} *\left(\boldsymbol{\omega}-\boldsymbol{\omega}_{b}+\mathbf{n}_{\boldsymbol{\omega}}\right), \\
\dot{\mathbf{v}} & =\mathbf{q} *\left(\mathbf{a}-\mathbf{a}_{b}+\mathbf{n}_{\mathbf{a}}\right) * \mathbf{q}^{-1}+\mathbf{g}, \\
\dot{\mathbf{x}} & =\mathbf{v} \\
\dot{\boldsymbol{\omega}}_{b} & =-\boldsymbol{\omega}_{b} / \tau_{\boldsymbol{\omega}}+\mathbf{n}_{\boldsymbol{\omega}_{b}}, \\
\dot{\mathbf{a}}_{b} & =-\mathbf{a}_{b} / \tau_{\mathbf{a}}+\mathbf{n}_{\mathbf{a}_{b}}, \\
\dot{\mathbf{w}} & =\mathbf{n}_{\mathbf{w}},
\end{aligned}
$$

where $\mathbf{q}$ is the unit quaternion that encodes the rotation of the body w.r.t. the inertial frame, $\mathbf{v}$ and $\mathbf{x}$ are, respectively, the UAV ground speed and position relative to the inertial frame, $\boldsymbol{\omega}_{b}$ and $\mathbf{a}_{b}$ are IMU bias, $\mathbf{w}$ is the wind speed, inputs $\boldsymbol{\omega}$ and a correspond, respectively, to biased noisy angular rate and body axis acceleration which are provided from an IMU, and $\mathbf{g}$ denotes the gravity field vector. We benefit here from the common quaternional form, whose basis properties, relations and advantages w.r.t. other representations are given in Appendix A. (1)-(5) are the standard IMU-based navigation dynamic where we suppose the biases follow a first order Gauss-Markov stochastic process in (4)-(5), with time constant $\tau_{\boldsymbol{\omega}}$ and $\tau_{\mathbf{a}}$, see. ${ }^{27,28}$ our constant wind model model (6) is justified in Section IV. We finally define

$$
\mathbf{n}=\left[\begin{array}{lllll}
\mathbf{n}_{\boldsymbol{\omega}}^{\top} & \mathbf{n}_{\mathbf{a}}^{\top} & \mathbf{n}_{\boldsymbol{\omega}_{b}}^{\top} & \mathbf{n}_{\mathbf{a}_{b}}^{\top} & \mathbf{n}_{\mathbf{w}}^{\top}
\end{array}\right]^{\top}
$$

that reflects the thermo-mechanical white and flicker noises on low-cost IMU direct measurements, the noise on low-cost IMU bias dynamics due to temperature effects and calibration errors, and the noise on wind dynamic.

\section{B. Measurements}

The UAV disposes of a large panel of low-cost sensors, giving our noisy measurements that we stack in

$$
\mathbf{y}=\left[\begin{array}{lllllll}
\mathbf{y}_{\mathbf{v}}^{\top} & \mathbf{y}_{\mathbf{x}}^{\top} & \mathbf{y}_{m}^{\top} & y_{b} & y_{p} & y_{\alpha} & y_{\beta}
\end{array}\right]^{\top},
$$

where $\mathbf{y}_{\mathbf{v}}$ and $\mathbf{y}_{\mathbf{x}}$ correspond, respectively, to the GPS velocity and position measurements, $\mathbf{y}_{m}$ comes from a magnetometer and $y_{b}$ from a barometer. An 1D Pitot static tube obtains the measurement $y_{p}$ of the airspeed in the forward $u$-direction since the tube is mounted on the UAV along the longitudinal axis, see Figure 1. The vanes (or pressure strips) provide measurements of AoA $\alpha$ and AoS $\beta$ which are represented in Figure 2 and calculated, respectively, as ${ }^{12}$

$$
\begin{aligned}
& \alpha=\tan ^{-1}\left(\frac{w_{a}}{u_{a}}\right), \\
& \beta=\sin ^{-1}\left(\frac{v_{a}}{\sqrt{u_{a}^{2}+v_{a}^{2}}}\right),
\end{aligned}
$$

where $\mathbf{v}_{a}=\left[\begin{array}{lll}u_{a} & v_{a} & w_{a}\end{array}\right]^{\top}$ denotes the airspeed and with $\beta$ measured parallel to the aircraft $w$-axis and thus does not contain a vertical airspeed component. The AoA represents the positive angle between the UAV wings and the airspeed, which is needed to generate lift, when the AoS relates to the rotation of the aircraft centerline from the airspeed, which decreases as the lateral acceleration increases during cornering, whereas in steady and level flight, airspeed $\mathbf{v}_{a}$ is commonly aligned with the $u$-axis, meaning $\beta$ is null. The equations 

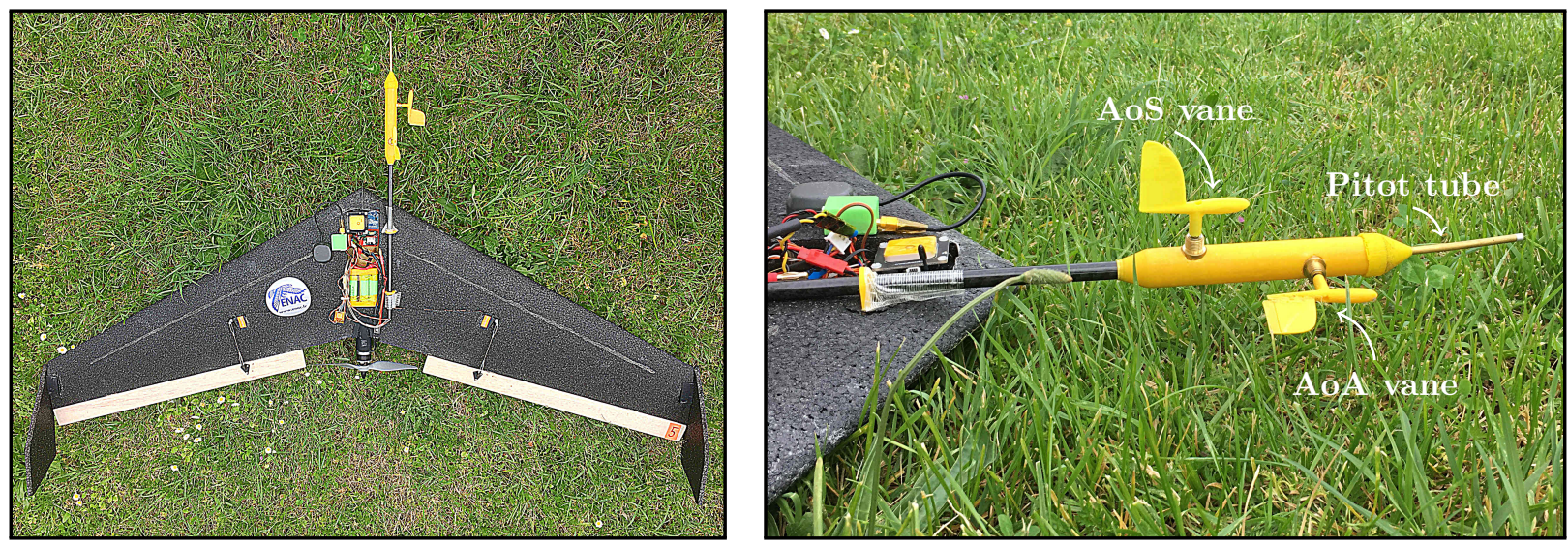

Figure 1. At left, the "Zagi", a fixed-wind UAV with an 1D Pitot static tube mounted along the longitudinal axis. At right, a zoom of the 1D Pitot static tube with two vanes that measure, respectively, Angle-of-Attack and Angle-of-Sideslip. The whole UAV and sensors are designed, manufactured and instrumented at the ENAC UAV laboratory.

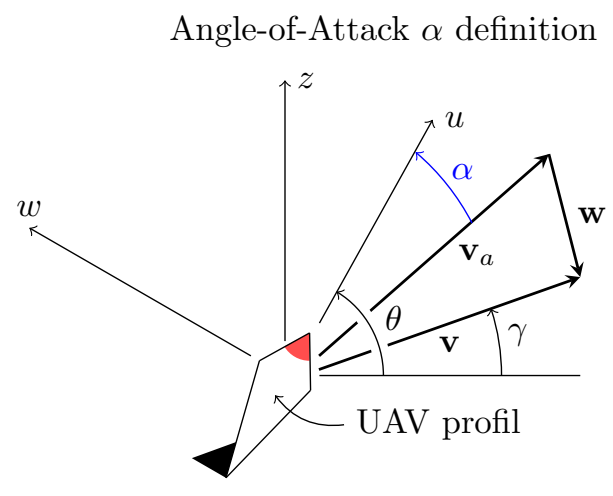

Angle-of-Sideslip $\beta$ definition

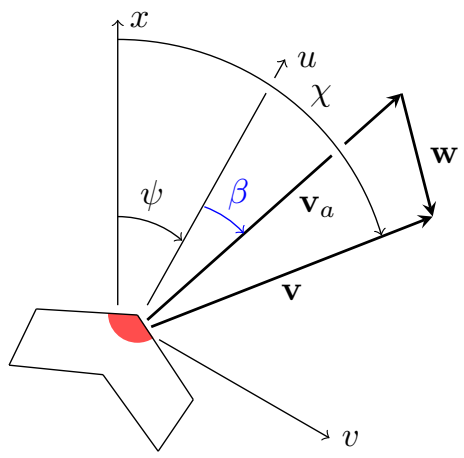

Figure 2. The wind triangle relationship between ground speed $\mathbf{v}$, airspeed $\mathbf{v}_{a}$ and wind w, projected, respectively, onto the vertical (at left) and horizontal (at right) planes, making in evidence the Angle-of-Attack $\alpha$ and the Angle-of-Sideslip $\beta$. The direction of the ground speed is specified using the course $\chi$, which represents the angle between the projection of $\mathrm{v}$ onto the $x y$-plane and north (i.e., the direction of travel relative to the Earth's surface) and the flight path $\gamma$ which is the angle between the $x y$-plane and v. The pitch $\theta$ moves the UAV nose up or down and heading (yaw) $\psi$ is the direction that the UAV is pointed. In the absence of wind, the difference between course and heading, the so-called crab-angle, is the Angle-of-Sideslip. 
then read

$$
\begin{aligned}
\mathbf{y}_{\mathbf{v}} & =\mathbf{v}+\mathbf{n}_{\mathbf{v}}, \\
\mathbf{y}_{\mathbf{x}} & =\mathbf{x}+\mathbf{n}_{\mathbf{x}}, \\
\mathbf{y}_{m} & =\mathbf{q}^{-1} * \mathbf{b} * \mathbf{q}+\mathbf{n}_{m} \\
y_{b} & =\mathbf{I}_{z} \mathbf{x}+n_{b}, \\
y_{p} & =\mathbf{I}_{u} \mathbf{v}_{a}+n_{p}, \\
y_{\alpha} & =\alpha+n_{\alpha}, \\
y_{\beta} & =\beta+n_{\beta},
\end{aligned}
$$

where $\mathbf{b}$ denotes the magnetic field vector, the line vectors $\mathbf{I}_{z}=\left[\begin{array}{lll}0 & 0 & 1\end{array}\right]$ and $\mathbf{I}_{u}=\left[\begin{array}{lll}1 & 0 & 0\end{array}\right]$ select, respectively, the third and first component of a vector and therefore extract the altitude in (14) and airspeed in the forward $u$-direction in (15). We finally stack all the sensor noises in

$$
\mathbf{n}_{\mathbf{y}}=\left[\begin{array}{lllllll}
\mathbf{n}_{\mathbf{v}}^{\top} & \mathbf{n}_{\mathbf{x}}^{\top} & \mathbf{n}_{m}^{\top} & n_{b} & n_{p} & n_{\alpha} & n_{\beta}
\end{array}\right]^{\top} .
$$

These measurements (11)-(17) arrive in discrete time at different sampling rates, which are usually much slower than the IMU measurement rate. The proposed filter in Section III matches particularly these asynchronous measurements since each of them can be treated independently or combined, and a loss of on of them (e.g., GPS) is straightforwardly handled by the filter. ${ }^{11}$

\section{Estimation Schemes}

There are various ways to tackle dynamical model (1)-(6) with measurement (8). Indeed, beyond the choice of a nonlinear filter (many possibilities exist such $\mathrm{as}^{20-24}$ ), there are important choices to be made. This is mainly due to the existence of algebraic equations that relate some variables, and particularly the so-called wind triangle relationship ${ }^{11,12}$

$$
\mathbf{v}=\mathbf{q} * \mathbf{v}_{a} * \mathbf{q}^{-1}+\mathbf{w}
$$

such that we should explicitly select two variables among $\mathbf{v}, \mathbf{v}_{a}$ and $\mathbf{w}$, while the remaining variable are implicitly defined. Let us consider in the sequel the two natural couples $(\mathbf{v}, \mathbf{w})$ and $\left(\mathbf{v}_{a}, \mathbf{w}\right)$ even if considering $\left(\mathbf{v}, \mathbf{v}_{a}\right)$ is also theoretically valid. With one couple selected, we can perform the wind estimation in two manners: treating the navigation problem first, which leads to a loosely coupled scheme; or in a tightly coupled approach, i.e., opting for a scheme that incorporates both the navigation and wind estimations. Thus, three main alternative approaches emerge, and we detail them in the rest of this section.

\section{Loosely Coupled Navigation and Wind Estimation}

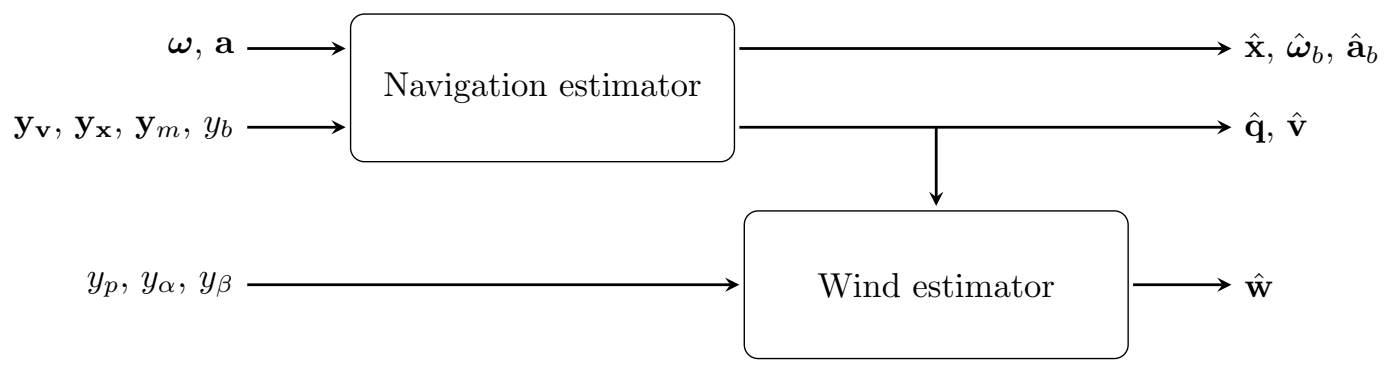

Figure 3. Loosely coupled approach. A first navigation estimator, typically an aided Inertial Navigation System, obtains estimates of navigation states. The estimated quaternion $\hat{\mathbf{q}}$ and ground speed $\hat{\mathbf{v}}$ are then used in a wind estimator building upon equation (19) to obtain the estimated wind $\hat{w}$.

This cascaded scheme consists of starting from the navigation estimator with dynamic (1)-(5) and measurements (11)-(14), to then launch a second estimator to obtain wind estimates by using the wind dynamic 
(6), the wind triangle (19), and the measurements (15)-(17), as depicted in Figure 3. The second estimator takes the required navigation estimates as input random variables with known mean and covariance to obtain $\hat{\mathbf{w}}$ through, e.g., Kalman filtering. ${ }^{16,19,29}$ The wind estimation depends strongly of the accuracy of the navigation estimates but have no impact on the navigation itself.

\section{Tightly Coupled Navigation and Wind Estimation with $\mathbf{v}$ and $\mathbf{w}$ as State Variables}

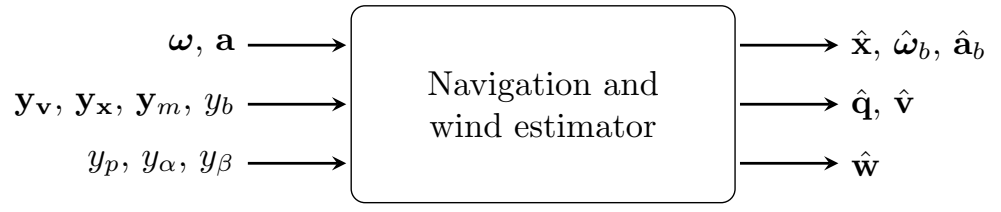

Figure 4. Tightly coupled approach. The only estimator obtains both navigation state and wind estimates, from all the available measurements, introducing thus correlation between estimated variables.

It is also possible to couple the whole model (1)-(6) when considered the whole measurement (8). The main interest of such approach compared to a cascaded scheme is the introduction of correlation between the wind estimates and navigation estimates, such that the airspeed related measurements (15)-(17) serve to improve the estimation of the navigation variables. We follow this approach in Section III, that are not been yet considered to the best of our knowledge.

\section{Tightly Coupled Navigation and Wind Estimation with $\mathbf{v}_{a}$ and $\mathbf{w}$ as State Variables}

The ideas are similar to the previous approach by substituting the airspeed $\mathbf{v}_{a}$ in place of the ground speed $\mathbf{v}$ in the state, an approach yet treated via Unscented Kalman Filter (UKF). ${ }^{15,17,18}$ We stress that for a same type of filter, the results between this approach and the above differ since: $i$ ) linearity is introduced in the 1D Pitot measurement (15) in place of GPS speed (11) which is rewritten as

$$
\mathbf{y}_{\mathbf{v}}=\mathbf{q} * \mathbf{v}_{a} * \mathbf{q}^{-1}+\mathbf{w}+\mathbf{n}_{\mathbf{v}}
$$

and $i$ ) error definitions differ strongly with the previous approach. Indeed, if we define $\mathbf{v}_{a}$ and $\mathbf{w}$ errors as Gaussian, the error on $\mathbf{v}$ is not Gaussian and conversely.

\section{Proposed MEKF-Wind Filter}

This section describe the proposed filter, which introduces correlation between the UAV state variables and wind estimates, via a tightly coupled scheme, see Section II.C.

\section{A. Retained Final Model}

We include both the ground speed and the wind in our state along with attitude, $\operatorname{position}^{\mathrm{a}}$, bias and wind, leading to the definitions of the state vector as

$$
\mathbf{z}=\left[\begin{array}{llllll}
\mathbf{q}^{\top} & \mathbf{v}^{\top} & \mathbf{x}^{\top} & \boldsymbol{\omega}_{b}^{\top} & \mathbf{a}_{b}^{\top} & \mathbf{w}^{\top}
\end{array}\right]^{\top},
$$

and state dynamic given in (1)-(6). We assume all the measurements (11)-(17) are available in discrete time and rewrite the $1 \mathrm{D}$ Pitot measurement (15) in function of the state variables as

$$
y_{p}=\mathbf{I}_{u}\left(\mathbf{q}^{-1} *(\mathbf{v}-\mathbf{w}) * \mathbf{q}\right)+n_{p}
$$

We choose to consider ground speed rather than airspeed since: $i$ ) it makes the retained model as an augmented model of the initial navigation problem, from which many works have been yet consecrated and

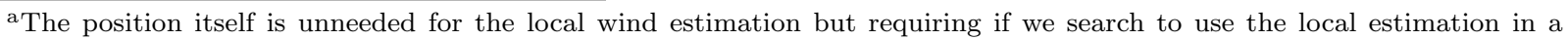
mapping scheme. ${ }^{6}$
} 
thus we can inspire of them and easily observe the consequences of our wind estimation on the navigation (not considering airspeed related measurements (15)-(17) lead directly to the original navigation problem); and $i i$ ) it let the GPS speed measurement linear and not attitude dependent in contrast with (20), which preserves a correct correction when attitude estimates are inaccurate. One could argue that the Pitot measurement (22) becomes nonlinear but: (22) is a scalar measurement whereas GPS gives three speed measurements (11); and we preserve linearity on the most confident measurement since in practice the noise on Pitot tube is much higher than on the GPS.

\section{B. Transformation of the Angle-of-Attack and Angle-of-Sideslip Measurements}

This section represents a major originality of the proposed filter. Indeed, the raw angle measurements (16)(17) are strongly non linear w.r.t. the state, that can lead to degraded performances. A naive approach to treat these measurements consists of considering the tangent of $y_{\alpha}$ and sinus of $y_{\beta}$, but it preserves a strong non-linearity. Inspired from works which convert range and angles in/from Cartesian coordinates for chemical processes estimation, ${ }^{25}$ robotics, ${ }^{?}$ or radar, ${ }^{30}$ we choose to transform these measurements in the following way, thus improving the filter performances, see Section V for an illustration.

Let us consider the two modified measurements

$$
\begin{aligned}
\tilde{y}_{\alpha} & =\mathbf{C v}_{a}, \\
& =\mathbf{C}\left(\mathbf{q}^{-1} *(\mathbf{v}-\mathbf{w}) * \mathbf{q}\right), \\
\tilde{y}_{\beta} & =\mathbf{v}_{a}^{\top} \mathbf{B} \mathbf{v}_{a}, \\
& =\left(\mathbf{q}^{-1} *(\mathbf{v}-\mathbf{w}) * \mathbf{q}\right)^{\top} \mathbf{B}\left(\mathbf{q}^{-1} *(\mathbf{v}-\mathbf{w}) * \mathbf{q}\right),
\end{aligned}
$$

where $\mathbf{C}$ and $\mathbf{B}$ are defined, respectively, as

$$
\begin{aligned}
& \mathbf{C}=\left[\begin{array}{lll}
\sin \left(y_{\alpha}\right) & 0 & -\cos \left(y_{\alpha}\right)
\end{array}\right] \text { and } \\
& \mathbf{B}=\left[\begin{array}{ccc}
\sin ^{2}\left(y_{\beta}\right) & 0 & 0 \\
0 & -\cos ^{2}\left(y_{\beta}\right) & 0 \\
0 & 0 & 0
\end{array}\right] .
\end{aligned}
$$

In the noise-free case, we have

$$
\begin{aligned}
\tilde{y}_{\alpha} & =\sin (\alpha) u_{a}-\cos (\alpha) w_{a}, \\
& =0, \\
\tilde{y}_{\beta} & =\sin ^{2}(\beta) u_{a}^{2}-\cos ^{2}(\beta) v_{a}^{2}, \\
& =\sin ^{2}(\beta) u_{a}^{2}-\left(1-\sin ^{2}(\beta)\right) v_{a}^{2}, \\
& =0,
\end{aligned}
$$

and we treat these modified measurements in our following proposed filter.

\section{Proposed Filter}

We choose to derive a Multiplicative EKF (M-EKF), ${ }^{21,23}$ called MEKF-Wind, for the considered estimation problem. The M-EKF is the standard algorithm in the inertial navigation systems industry. Albeit increasingly replaced with the UKF, it remains computationally more efficient, ${ }^{31}$ and the name Multiplicative is coined as the quaternion-based error is computed using a quaternion product $*$ instead of subtraction. Specifically, the three-dimensional error vector $\boldsymbol{\epsilon}_{\mathbf{q}}$ obtained from the quaternion error after using a small angle approximation is maintained in the error state, resulting in a minimal representation that is safe to use in calculating the covariance. In the following, we make use a continuous-discrete representation ${ }^{11}$ for our implementation of the MEKF-Wind.

The filter consists of two steps: propagation and update, which are summarized in Algorithm $1^{\mathrm{b}}$. During prediction (lines 1 and 2), the filter produces estimates of the current state variables, along with their

\footnotetext{
${ }^{\mathrm{b}}$ A more sophisticated integration (line 1) with exact quaternion propagation or Runge-Kunta method can also be effected. ${ }^{32}$ At the end of this state propagation, we additionally enforce to the quaternion estimate $\hat{\mathbf{q}}$ to have a unit norm.
} 


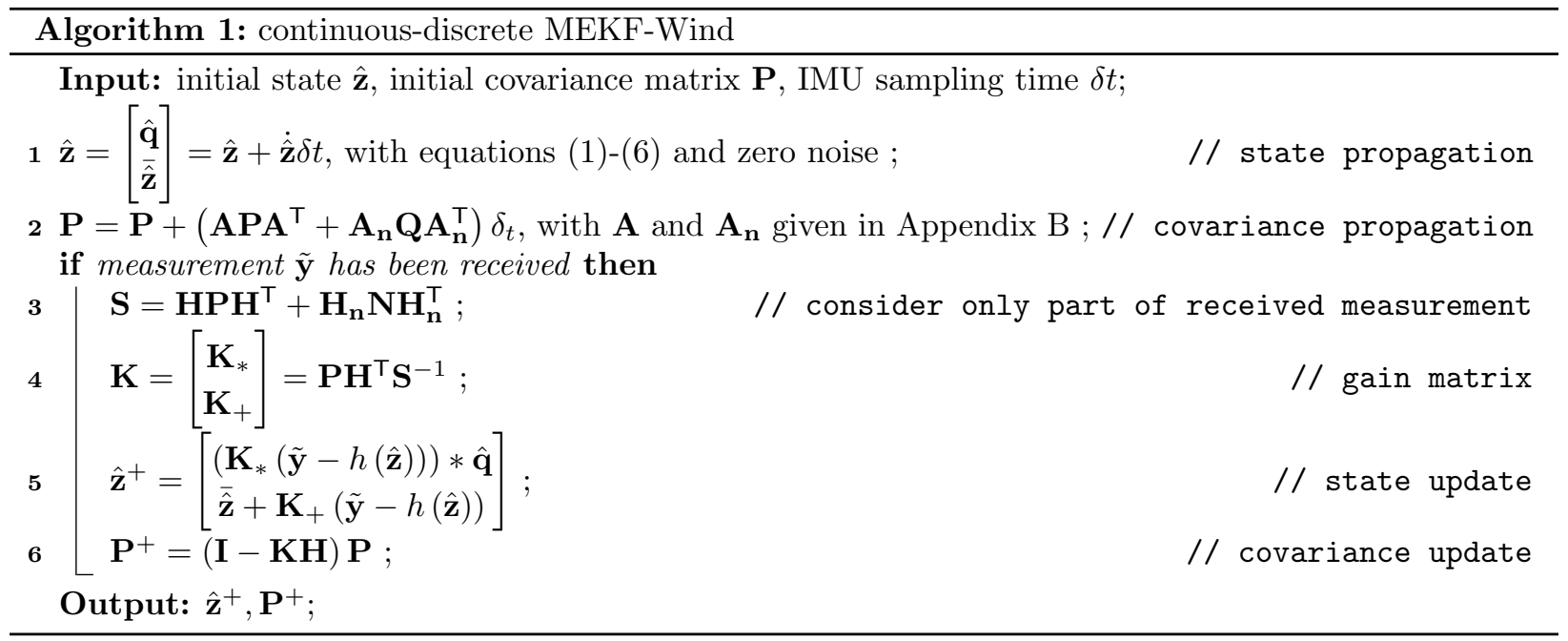

uncertainties, and we move the mathematical machinery to Appendix B. In the update step (lines 3 to 6 ), the gain matrix $\mathbf{K}$ is divided into $\mathbf{K}_{*}$ for the quaternion error and $\mathbf{K}_{+}$for the remaining state errors. The previous treatment of the anglular measurements results in the definition of the transformed measurement $\tilde{\mathbf{y}}$ and transformed measurement function $h($.$) , respectively, as$

$$
\begin{aligned}
& \tilde{\mathbf{y}}=\left[\begin{array}{lllllll}
\mathbf{y}_{\mathbf{v}}^{\top} & \mathbf{y}_{\mathbf{x}}^{\top} & \mathbf{y}_{m}^{\top} & y_{b} & y_{p} & -\mathcal{E}\left\{\tilde{y}_{\alpha}\right\} & -\mathcal{E}\left\{\tilde{y}_{\beta}\right\}
\end{array}\right]^{\top}, \\
& h(\mathbf{z})=\left[\begin{array}{c}
\mathbf{v} \\
\mathbf{x} \\
\mathbf{q}^{-1} * \mathbf{b} * \mathbf{q} \\
\mathbf{I}_{z} \mathbf{x} \\
\mathbf{I}_{u} \mathbf{v}_{a} \\
\mathbf{C v}_{a} \\
\mathbf{v}_{a}^{\top} \mathbf{B} \mathbf{v}_{a}
\end{array}\right]
\end{aligned}
$$

where $\mathbf{C}, \mathbf{B}$ are given in (25)-(26) and let the observation matrix $\mathbf{H}$ be defined as

$$
\mathbf{H}=\left[\begin{array}{cccccc}
\mathbf{0} & \mathbf{I} & \mathbf{0} & \mathbf{0} & \mathbf{0} & \mathbf{0} \\
\mathbf{0} & \mathbf{0} & \mathbf{I} & \vdots & \vdots & \vdots \\
\hat{\mathbf{R}}_{\mathbf{q}}^{\top}(\mathbf{b})_{\times} & \vdots & \mathbf{0} & \vdots & \vdots & \vdots \\
\mathbf{0} & \mathbf{0} & \mathbf{I}_{z} & \vdots & \vdots & \mathbf{0} \\
\mathbf{I}_{u} \hat{\mathbf{R}}_{\mathbf{q}}^{\top}(\hat{\mathbf{v}}-\hat{\mathbf{w}})_{\times} & \mathbf{I}_{u} \hat{\mathbf{R}}_{\mathbf{q}}^{\top} & \mathbf{0} & \vdots & \vdots & -\mathbf{I}_{u} \hat{\mathbf{R}}_{\mathbf{q}}^{\top} \\
\mathbf{C} \hat{\mathbf{R}}_{\mathbf{q}}^{\top}(\hat{\mathbf{v}}-\hat{\mathbf{w}})_{\times} & \mathbf{C} \hat{\mathbf{R}}_{\mathbf{q}}^{\top} & \vdots & \vdots & \vdots & -\mathbf{C} \hat{\mathbf{R}}_{\mathbf{q}}^{\top} \\
2 \hat{\mathbf{v}}_{a} \mathbf{B} \hat{\mathbf{R}}_{\mathbf{q}}^{\top}(\hat{\mathbf{v}}-\hat{\mathbf{w}})_{\times} & 2 \hat{\mathbf{v}}_{a} \mathbf{B} \hat{\mathbf{R}}_{\mathbf{q}}^{\top} & 0 & 0 & 0 & -2 \hat{\mathbf{v}}_{a} \mathbf{B} \hat{\mathbf{R}}_{\mathbf{q}}^{\top}
\end{array}\right]
$$

with $\hat{\mathbf{R}}_{\mathbf{q}}$ the (estimated) direction cosine matrix corresponding to the rotation operator $\hat{\mathbf{q}} *(). * \hat{\mathbf{q}}^{-1},(.)_{\times}$the skew symmetric operator representing cross products as matrix multiplications, and where the measurement transformation introduces the bias $\mathcal{E}\left\{\tilde{y}_{\alpha}\right\}$ and $\mathcal{E}\left\{\tilde{y}_{\beta}\right\}$, which are null in first order approximation. Finally, $\mathbf{Q}$ and $\mathbf{N}$ encodes, respectively, the covariances of the dynamic process noise $\mathbf{n}$ and measurement noise $\mathbf{n}_{\mathbf{y}}$, typically diagonal matrices, and $\mathbf{H}_{\mathbf{n}}$ is the Jacobian of $\tilde{\mathbf{y}}$ w.r.t. the measurement noise defined as the diagonal matrix

$$
\mathbf{H}_{\mathbf{n}}=\operatorname{diag}\left(\mathbf{1}, \mathbf{1}, \mathbf{1}, 1,1, \frac{\partial \tilde{y}_{\alpha}}{\partial n_{\alpha}}, \frac{\partial \tilde{y}_{\beta}}{\partial n_{\beta}}\right) \text {, with }
$$




$$
\begin{aligned}
& \frac{\partial \tilde{y}_{\alpha}}{\partial n_{\alpha}}=-\left[\begin{array}{lll}
\cos (\alpha) & 0 & \sin (\alpha)
\end{array}\right] \hat{\mathbf{v}}_{a}, \\
& \frac{\partial \tilde{y}_{\beta}}{\partial n_{\beta}}=[-\sin (2 \beta) \quad 0 \quad \sin (2 \beta)] \operatorname{diag}\left(\hat{\mathbf{v}}_{a}\right) \hat{\mathbf{v}}_{a},
\end{aligned}
$$

and where diag (.) converts its vector arguments to a diagonal matrix with the vectors aligned on the main diagonal. We let the getting of bias and Jacobians of $\tilde{y}_{\alpha}$ and $\tilde{y}_{\beta}$, leading to both $\mathbf{H}$ and $\mathbf{H}_{\mathbf{n}}$, in Appendix C.

\section{Noise Tuning and Wind Statistics}

We must provide our filter with some values about the noise covariances both for the dynamic and measurement models, affecting both filter convergence and performances. Let us divide the various noises into three classes: i) navigation noise incorporating the IMU random process, IMU and navigation measurements noises, i.e, $\mathbf{n}_{\boldsymbol{\omega}_{b}}, \mathbf{n}_{\mathbf{a}_{b}}, \mathbf{n}_{\boldsymbol{\omega}}, \mathbf{n}_{\mathbf{a}}, \mathbf{n}_{\mathbf{v}}, \mathbf{n}_{\mathbf{x}}, \mathbf{n}_{m}$ and $n_{b} ;$ ii) airspeed sensor noise containing the noises $n_{p}, n_{\alpha}$ and $n_{\beta}$ of the airspeed related measurements (15)-(17); and iii) wind process noise $\mathbf{n}_{\mathbf{w}}$. The two first classes are discussed in the following subsection, the third one is based on real data and discussed in the following subsection.

\section{A. Sensor Noise Identification}

We consider in this subsection the parameter tuning of the navigation noise and airspeed sensor noise. Classical techniques and sensor characteristics yield the noise covariances for the IMU and measurements (11)-(14), which can be done simultaneously to bias time constant identification. ${ }^{27,28}$ Concerning then the airspeed related measurements (15)-(17), we obtain a first covariance of the static noise when the UAV is motionless. We then have the noise due to non-perfect calibration in wind tunnel and inherent noise on the instruments, that are inaccurate for small airspeed $\mathbf{v}_{a}<10 \mathrm{~m} / \mathrm{s}$, i.e., before and in takeoff. More precisely, we consider only the airspeed related measurements (15)-(17) when the flight is yet established, which is equivalent to put an infinite noise covariance before flight establishment, that additionally avoids trouble due to wrong initialization.

\section{B. Wind Statistics}

We concentrate now on the justification of our wind dynamical model (6) and then propose a simple wind noise tuning, by analyzing data provided by the Midi-Pyrénées Observatoryc ${ }^{\mathrm{c}}$

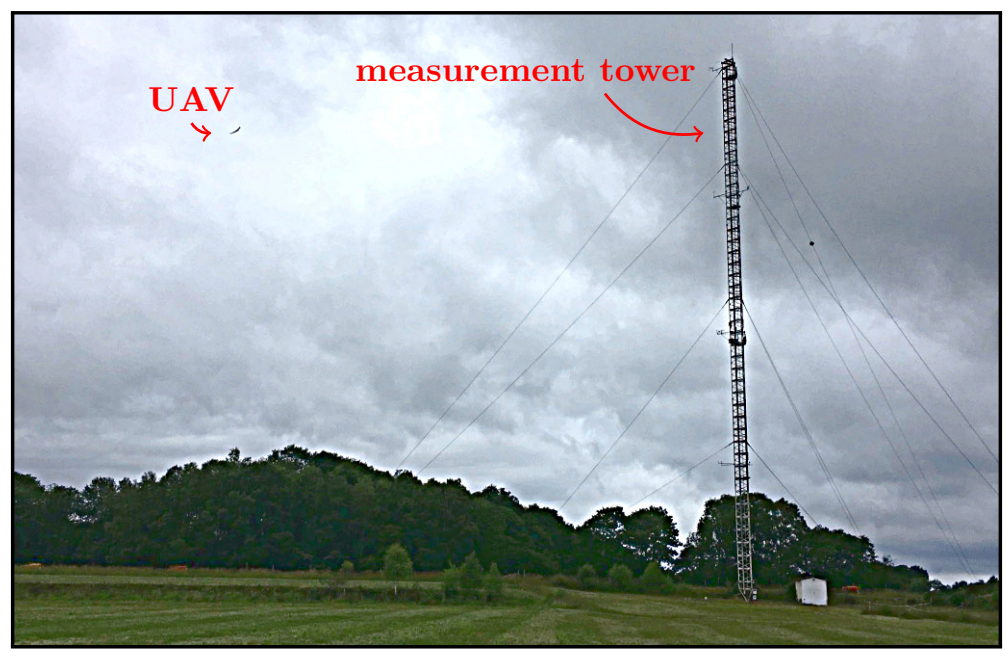

Figure 5. 60-m measurement tower, equipped with CSAT3 and GILL sonic anemometers at $30 \mathrm{~m}, 45 \mathrm{~m}$ and $60 \mathrm{~m}$, which provide accurate $3 \mathrm{D}$ wind measurements at $10 \mathrm{~Hz}$ sampling rate.

\footnotetext{
${ }^{c}$ http://www.obs-mip.fr/
} 


\section{Empirical Justification of the Wind Dynamic (6)}

The Midi-Pyrénées Observatory land in Campistrous ${ }^{\mathrm{d}}$ disposes of a $60-\mathrm{m}$ measurement tower which provides wind measurements in a large flat area, see Figure 5. The wind sensors consist of sonic anemometers CSAT3 and GILL, which obtain accurately 3D wind estimates equivalent to a ground truth with high sampling rate of $10 \mathrm{~Hz}$ at three different altitudes: $30 \mathrm{~m}, 45 \mathrm{~m}$ and $60 \mathrm{~m}$. We plot data acquired on 27 April 2017, give a half-hour zoom representing the wind variations for the time of a UAV flight in Figure and wind statistics about these data in, respectively, Figure 6, Figure 7 and Table 1.
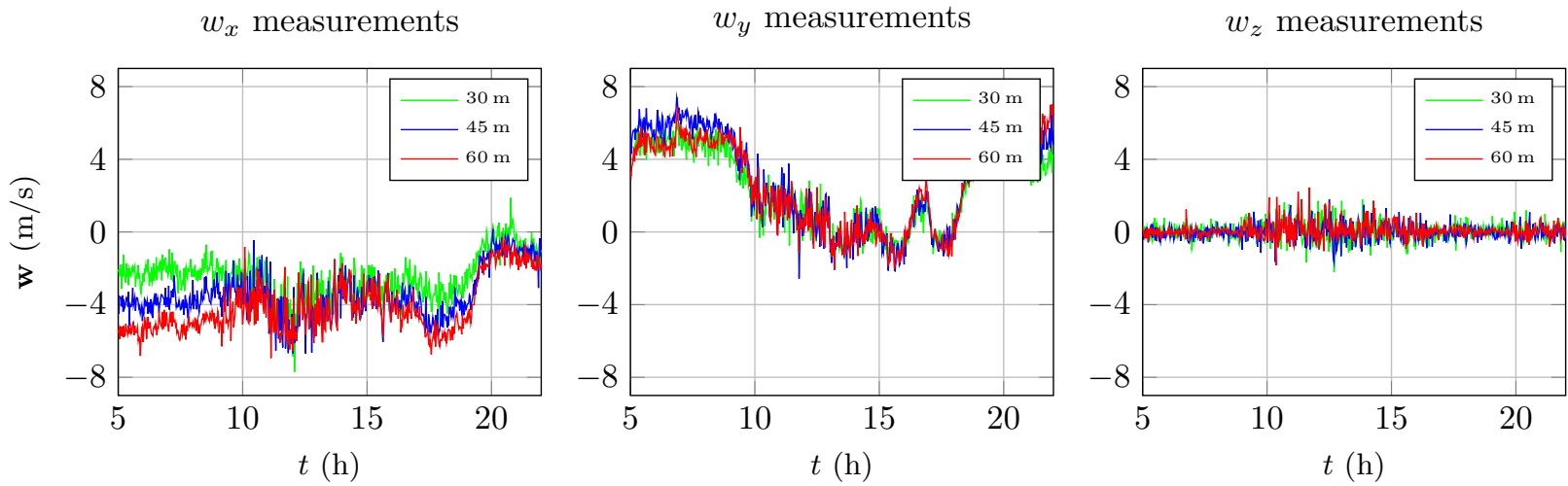

Figure 6. Wind speed obtained by the measurement tower at $30 \mathrm{~m}, 45 \mathrm{~m}$ and $60 \mathrm{~m}$ for each wind components, as function of time, between $5 \mathrm{am}(t=5 \mathrm{~h})$ and $10 \mathrm{pm}$, on 27 April 2017, a relatively cloudy day. The correlation of wind w.r.t. altitude appears evident, and we observe weak correlation between wind components.

From these data, we observe clearly weak correlation between each component of the wind in Figure 6 and in the right part of Table 1, i.e., each wind component follows a relatively independent dynamic, leading to choosing a diagonal covariance of the wind noise process. We also notice a strong correlation of the wind speed w.r.t. the altitude for $w_{x}$ and particularly for $w_{y}$. However, defining a static wind profile or a Dryden model ${ }^{13}$ appears challenging as the factors mapping the wind at different altitude vary, and this becomes unfeasible for the very short period time of a flight ${ }^{33}$ as in Figure 7, therefore justifying our random process model.

$w_{x}$ measurements

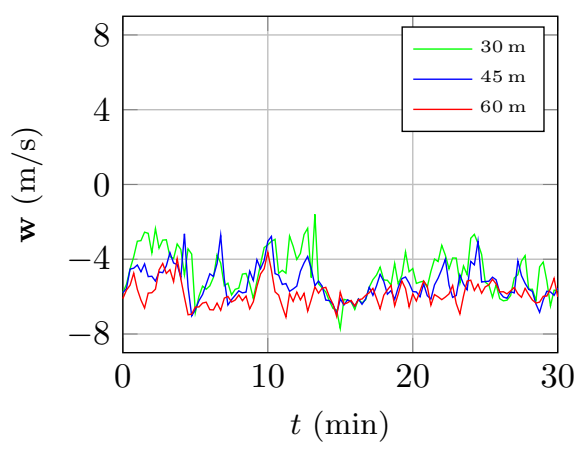

$w_{y}$ measurements

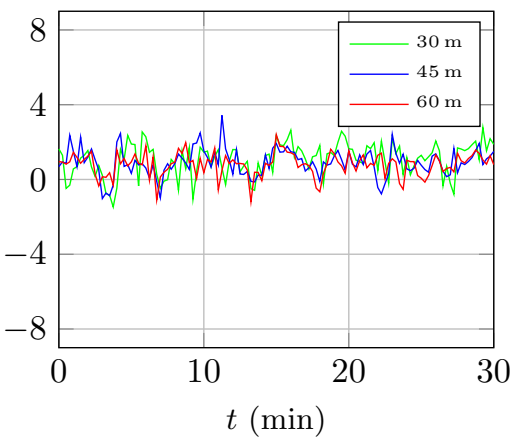

$w_{z}$ measurements

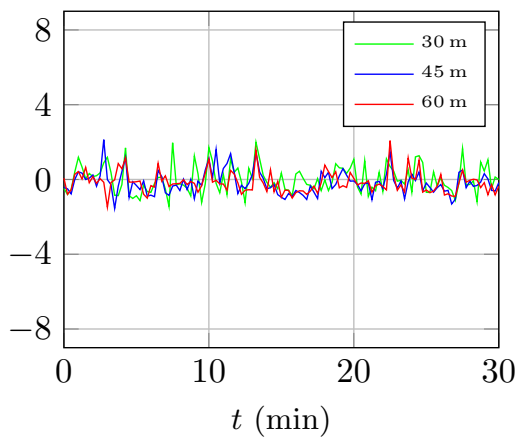

Figure 7. Wind speed obtained by the measurement tower at $30 \mathrm{~m}, 45 \mathrm{~m}$ and $60 \mathrm{~m}$ for each wind components, as function of time, between $1 \mathrm{pm}$ and one half past $1 \mathrm{pm}$, on 27 April 2017. We clearly see that one can not correlate the measurements at different altitudes through linear regression.

\footnotetext{
${ }^{\mathrm{d}}$ The site is located in Campistrous, France, at N $43^{\circ} 7^{\prime} 464^{\prime \prime} \mathrm{E} 0^{\circ} 22^{\prime} 98^{\prime \prime}$.
} 


\begin{tabular}{|c|c|c|c|c|c|}
\hline altitude measurement & $45 \mathrm{~m}$ & $60 \mathrm{~m}$ & wind component & $w_{y}$ & $w_{z}$ \\
\hline $30 \mathrm{~m}$ & 0.93 & 0.94 & $w_{x}$ & -0.14 & 0.31 \\
\hline $45 \mathrm{~m}$ & - & 0.96 & $w_{y}$ & - & 0.13 \\
\hline
\end{tabular}

Table 1. Correlation factors between the wind speed at different altitudes (at left) and different wind components (at right), with high values w.r.t. the different altitudes and weak values w.r.t. the different components.

\section{Tuning of Wind Process Noise Covariance}

The wind model being (at least partially) justified, the parameters are still to be tuned, i.e. the covariance of $\mathbf{n}_{\mathbf{w}}$. Compared to the measurement tower, the UAV is moving. When the latter is slowly flying or unmoving, it has difficulty to precisely estimate the airspeed and thus the wind, consequently we freeze the wind dynamic with zero noise covariance, i.e., $\mathbf{n}_{\mathbf{w}}=\mathbf{0}$, such that the wind converge to a static value (which differs from imposing $\mathbf{w}=\mathbf{0}$ ). During flight, with $\mathbf{n}_{\mathbf{w}} \neq \mathbf{0}$, an instinctive tunning would to increase the noise as the UAV speed increases since wind is also spatially distributed. ${ }^{33,34}$ However, this appears complicated to handle, and we prefer to have two noise covariance values: one null when the airspeed related measurements are inactive (i.e., before and during takeoff, see Section IV.A); one non-null value otherwise, and let a numerically and more sophisticated tuning for future research. A practical consequence of such tunning is to have a wind fixed to its initial values until flight establishment (where there are no reason to insert correlation between wind and another variables in the initial $\mathbf{P}$ ).

\section{Simulation Results}

\section{A. Simulation Setting}

A simulation study is conducted to test the proposed filter when an explicit truth is available. We consider the flight simulation environment proposed by the Paparazzi ${ }^{\mathrm{e}}$ autopilot system ${ }^{35}$ to compare the three following approaches that assumes the same dynamic (1)-(6): i) $\mathbf{v}_{a}$-UKF, an UKF which incorporates Euler angles and the airspeed (19) in place of the quaternion and the ground speed, and considers the direct measurement (8), which is similar to the filter of Rhudy et. al; $\left.{ }^{17} i i\right), \mathbf{v}-\mathrm{UKF}$, a novel UKF which incorporates Euler angles, ground speed, and considers the proposed measurement (29); and iii) MEKF-Wind, which incorporates the state vector (21) and considers the transformed measurement (29) where we set bias to zero, improving thus time-efficiency without decreasing performances in practical scenarios ${ }^{\mathrm{f}}$.

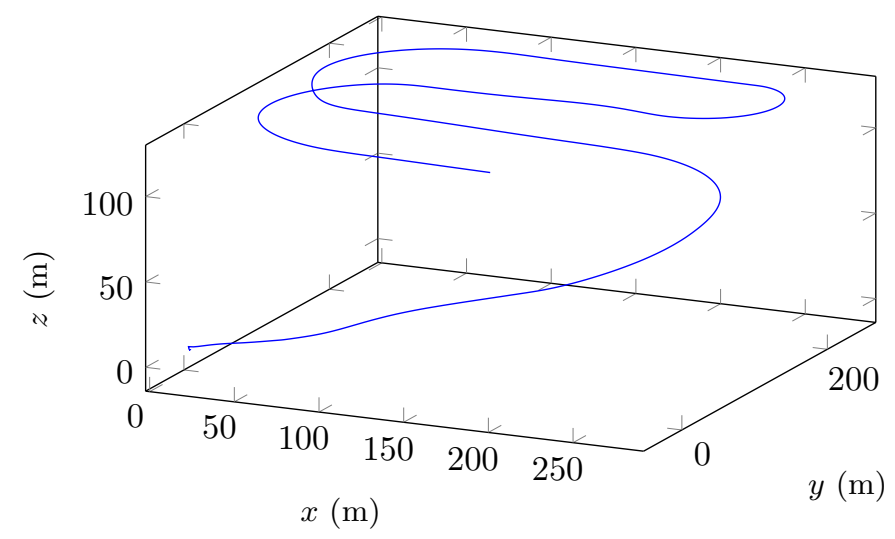

Figure 8. Paparazzi simulation trajectory, starting close to the origin. Flight parameters have standard values, constant wind speed is $w_{x}=4 \cos (30) \mathrm{m} / \mathrm{s}, w_{y}=4 \sin (30) \mathrm{m} / \mathrm{s}$ and $w_{z}=0.5 \mathrm{~m} / \mathrm{s}$. The trajectory represents a starting $300 \mathrm{~m} \times 200 \mathrm{~m}$-box survey of $150 \mathrm{~s}$, with soaring, curves and straight lines.

The simulation flight consists of $150 \mathrm{~s}$ of a starting $300 \mathrm{~m} \times 200 \mathrm{~m}$-box survey, where we combine standard flight parameters with an artificial constant wind of $4 \mathrm{~m} / \mathrm{s}$ in $x y$-plan, $0.5 \mathrm{~m} / \mathrm{s}$ in the $z$-axis and with direction

ehttp://wiki.paparazziuav.org/.

${ }^{\mathrm{f}}$ Indeed, it appears in simulation that the unbiased measurements increase performances only with unrealistic weak noise. 


\begin{tabular}{c|c|c|c|c|c|c|c|c} 
Parameter & $\sigma_{\boldsymbol{\omega}}\left(\mathrm{rad} / \mathrm{s}^{\frac{3}{2}}\right)$ & $\sigma_{\mathbf{a}}\left(\mathrm{m} / \mathrm{s}^{\frac{5}{2}}\right)$ & $\sigma_{\boldsymbol{\omega}_{b}}\left(\mathrm{rad} / \mathrm{s}^{\frac{3}{2}}\right)$ & $\sigma_{\mathbf{a}_{b}}\left(\mathrm{~m} / \mathrm{s}^{\frac{5}{2}}\right)$ & $\sigma_{\mathbf{w}}\left(\mathrm{m} / \mathrm{s}^{\frac{3}{2}}\right)$ & $\sigma_{\mathbf{v}}(\mathrm{m} / \mathrm{s})$ & $\sigma_{\mathbf{x}}(\mathrm{m})$ \\
\hline Value & $3 \cdot 10^{-3}$ & $3 \cdot 10^{-2}$ & $3 \cdot 10^{-4}$ & $10^{-2}$ & $10^{-2}$ & $10^{-1}$ & 1 \\
Parameter & $\sigma_{m}(\mathrm{~T})$ & $\sigma_{b}(\mathrm{~m})$ & $\sigma_{p}(\mathrm{~m} / \mathrm{s})$ & $\sigma_{\alpha}(\mathrm{rad})$ & $\sigma_{\beta}(\mathrm{rad})$ & $\tau_{\boldsymbol{\omega}}(\mathrm{rad} / \mathrm{s})$ & $\tau_{\mathbf{a}}\left(\mathrm{m} / \mathrm{s}^{2}\right)$ \\
\hline Value & $10^{-5}$ & 1 & $3 \cdot 10^{-1}$ & $10^{-1}$ & $10^{-1}$ & $8 \cdot 10^{2}$ & $10^{3}$
\end{tabular}

Table 2. Parameter values for the simulations, with high-moderate noise.

of $30^{\circ}$ in $x y$-plan. The noises are assumed independent, zero mean and we define their standard deviation to moderate-high realistic values given in Table 2 with the time constants. We then recover data from the simulation system, whose resulting trajectory is displayed in Figure 8. We initialize the three filters with the raw estimates corresponding of the first measurements, specifically for the wind we use a direct method. ${ }^{13}$ We set a moderate uncertainty for the wind covariance in $\mathbf{P}$ and a small uncertainty for the remaining state to be coherent with the conditions before real takeoff, i.e., being more confident for the UAV states than for the wind velocity.

\section{B. Simulation Results}

We give these noisy measurements to the three filters and plot the root square error w.r.t. the wind speed in Figure 9. We clearly observe the accuracy of MEKF-Wind and $\mathbf{v}$-UKF compared to $\mathbf{v}_{a}$-UKF once flight establishment, see Section II.C for justifications. The three filters keep a non null error since they suppose wind is quick varying as in practice (i.e., a high value for $\mathbf{n}_{\mathbf{w}}$ ) and noise is set moderate-high.

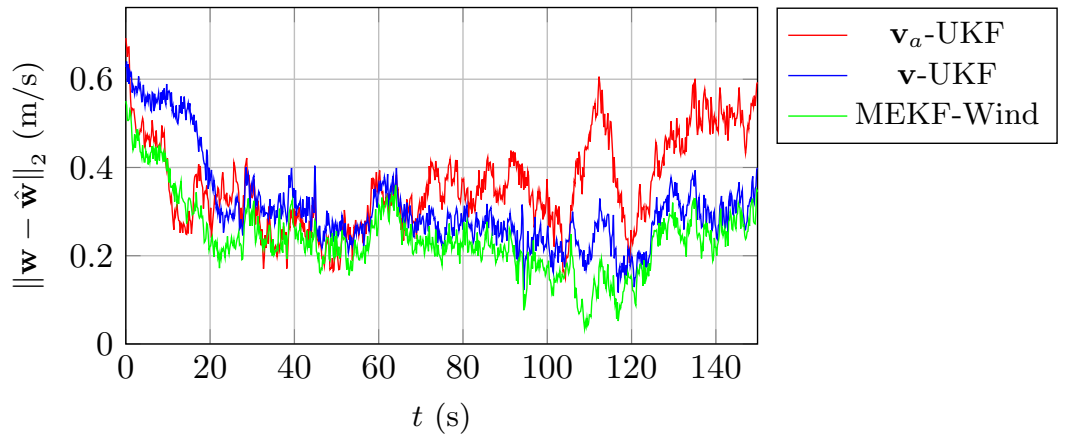

Figure 9. Root square error on the wind speed, as a function of time, with realistic noisy measurements. MEKF-Wind obtain best results than $\mathrm{v}-\mathrm{UKF}$ and $\mathrm{v}_{a}$-UKF once flight establishment.

We then run 200 Monte-Carlo simulations for this setting to compare the filter statistic performances, which are indicated in Table 3. This confirms the superiority of the MEKF-Wind, which better estimates attitude, position and wind speed, and outperforms $\mathbf{v}$-UKF particularly for the attitude, that is certainly due to its quaternional error representation. The bias errors are similar for the three filters and the EstimatedSTandard Deviation of MEKF-Wind are coherent with the Root Mean Square Error, which indicates that the filter is not overconfident. An unscented version of the proposed filter, which makes use of an identical quaternional error representation, ${ }^{24}$ would probably obtains similar results. However, one major advantage of the MEKF-Wind over such unscented filters is its computational time-efficiency, particularly important with the relatively large size of the space-state. ${ }^{31}$

We finally stress that the initial uncertainty and noise covariance values affects the convergence of the estimates for all methods, which indicates the need of tuning numerically the filter parameters. However, with different initializations and realistic covariances, the tendency and observations are identical as for the indicated results. 


\begin{tabular}{c|ccc|ccc}
\multirow{2}{*}{ State component } & \multicolumn{4}{|c}{ RMSE } & \multicolumn{3}{c}{ Estimated-STD } \\
& MEKF & $\mathbf{v}$-UKF & $\mathbf{v}_{a}$-UKF & MEKF & $\mathbf{v}$-UKF & $\mathbf{v}_{a}$-UKF \\
\hline Euler angles $\left(^{\circ}\right)$ & 2.0 & 5.5 & 6.0 & 2.5 & 1.7 & 1.7 \\
$\mathbf{v}(\mathrm{m} / \mathrm{s})$ & $3.1 \cdot 10^{-2}$ & $5.1 \cdot 10^{-2}$ & - & $9.0 \cdot 10^{-2}$ & $8.8 \cdot 10^{-2}$ & - \\
$\mathbf{x}(\mathrm{m})$ & $6.3 \cdot 10^{-2}$ & $1.0 \cdot 10^{-1}$ & $1.2 \cdot 10^{-1}$ & $5.1 \cdot 10^{-1}$ & $5.1 \cdot 10^{-1}$ & $5.1 \cdot 10^{-1}$ \\
$\boldsymbol{\omega}_{b}(\mathrm{rad} / \mathrm{s})$ & $5.1 \cdot 10^{-3}$ & $4.9 \cdot 10^{-3}$ & $5.1 \cdot 10^{-3}$ & $1.2 \cdot 10^{-2}$ & $5.2 \cdot 10^{-2}$ & $5.1 \cdot 10^{-2}$ \\
$\mathbf{a}_{b}\left(\mathrm{~m} / \mathrm{s}^{2}\right)$ & $8.0 \cdot 10^{-2}$ & $8.0 \cdot 10^{-2}$ & $8.1 \cdot 10^{-2}$ & $2.0 \cdot 10^{-2}$ & $2.1 \cdot 10^{-2}$ & $2.0 \cdot 10^{-2}$ \\
$\mathbf{w}(\mathrm{m} / \mathrm{s})$ & $1.8 \cdot 10^{-1}$ & $2.2 \cdot 10^{-1}$ & $2.7 \cdot 10^{-1}$ & $2.4 \cdot 10^{-1}$ & $2.4 \cdot 10^{-1}$ & $2.0 \cdot 10^{-1}$
\end{tabular}

Table 3. Root Mean Square Error (RMSE) w.r.t. the state variables and Estimated-STandard Deviation (Estimated-STD) given by the estimated covariance $P$. The values are averaged over 200 Monte-Carlo simulations and Euler angles error is indicated rather than quaternion error. MEKF-Wind outperforms both v-UKF and $\mathbf{v}_{a}-\mathrm{UKF}$, with coherent standard deviation estimates.

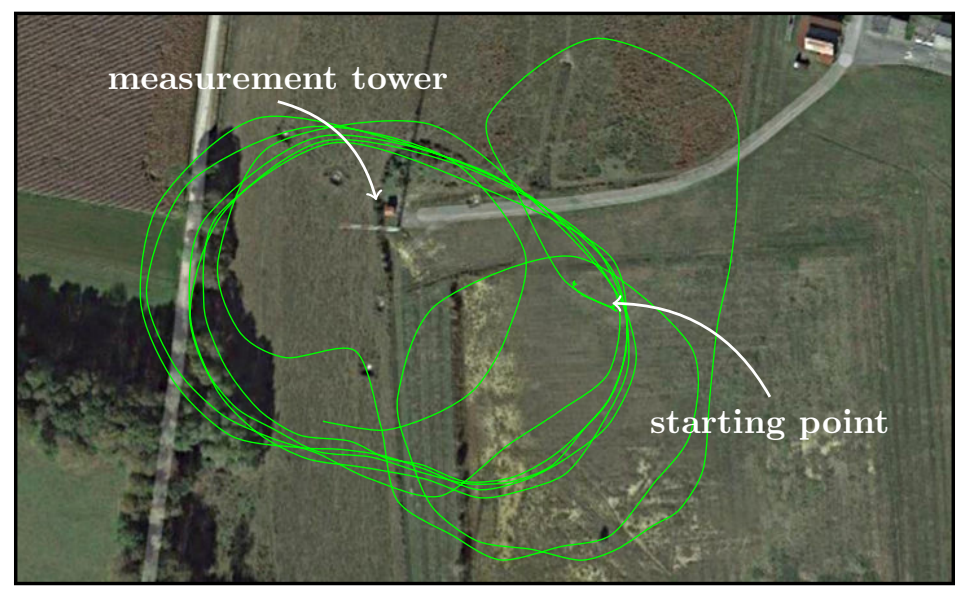

Figure 10. Flight trajectory in the Midi-Pyrénées Observatory land (France), consisting of ovals around the 60-m measurement tower, during $13 \mathrm{~min}$. Particular intentions are made to obtain a trajectory close to the measurement tower.

\section{Application on Flight Experiment}

\section{A. Experimental Setting}

An experimental study is necessary for validating such wind estimation methods since the discrepancy between real and simulation flights is particularly important for the wind behavior, which can includes, e.g., gusts or thermal ascents. ${ }^{34}$ Specific interest is to obtain real-time wind data as close as the wind experimented by the aircraft. Toward this aim, we conduct the flight experiment in the Midi-Pyrénées Observatory, see Figure 10, where we yet obtain data from the 60-m measurement tower described at Section IV. The validation data at altitudes $30 \mathrm{~m}^{\mathrm{g}}$ are plotted in Figure 11 for the flight time and as remarked in Section IV, since fitting a wind profile (e.g., a power law w.r.t. the altitude) for such very short-period time is irrelevant, data acquired here are clearly accurate compared to, e.g., a simple portable ground weather station.

ENAC UAV laboratory ${ }^{\mathrm{h}}$ has developed a fixed-wind UAV so-called "Zagi", which is a mini UAV of wingspan $1.1 \mathrm{~m}$ for $0.5 \mathrm{~kg}$ visible on Figure 1. Its onboard sensor consists of an Apogee v1.00 PCB flight controller with the Paparazzi autopilot, GPS RTK and HMC5983 magnetometers. We mount a self-made 1D Pitot static tube, connected to HCLA12X5EB pressure sensor, on the nose of the aircraft along the longitudinal axis, which comports both AoA and AoS vanes, each associated with an analog-to-digital converter

\footnotetext{
g Anemometers at $45 \mathrm{~m}$ and $60 \mathrm{~m}$ were breakdown during flight. We schedule new experiments once sensors are fixed and consider these new experiments flights in the final version of the paper.

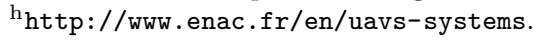


and the whole sensor suite is yet calibrated in wind tunnel. Data are finally logged on a micro SD card with a light Real-Time Operating System called ChibiOS.

Flight experiments are realized on 6 June 2017, starting at 36 past 11 (am), the weather is cloudy and wind is moderate-high, as confirmed by the measurement tower in Figure 11. We define the flight trajectory as ovals around the measurement tower at stable altitude and search to keep close to the measurement power, to then justifiably compare the wind estimates at the measurement tower and at the UAV locations.

\section{B. Experimental Results}

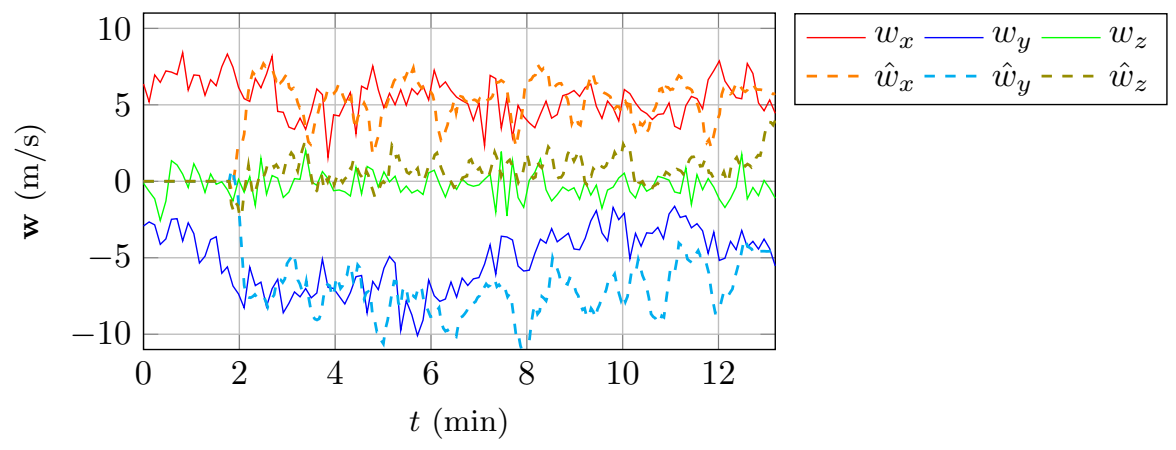

Figure 11. Wind speed from the measurement tower at $30 \mathrm{~m}$ and wind estimates of the MEKF-Wind, as function of time. Null estimates at the two first minutes are a consequence of the tuning of noise parameters.

Giving the raw acquired measurements to the MEKF-wind, the filter replays the flight and obtains wind estimates plotted in Figure 11. As we activate the airspeed measurements once takeoff is effected (see Section IV.B), we have null estimates at the two first minutes. We see that the wind estimates matches the wind as measured by the measurement tower in mean, while none of these estimates closely match the "ground truth". This comes as no surprise since the measurement tower is measuring wind at a different locations, and local turbulence could cause differences between the wind speed measured at different points and the values of the tuning parameters impact the dynamics of the wind estimates.
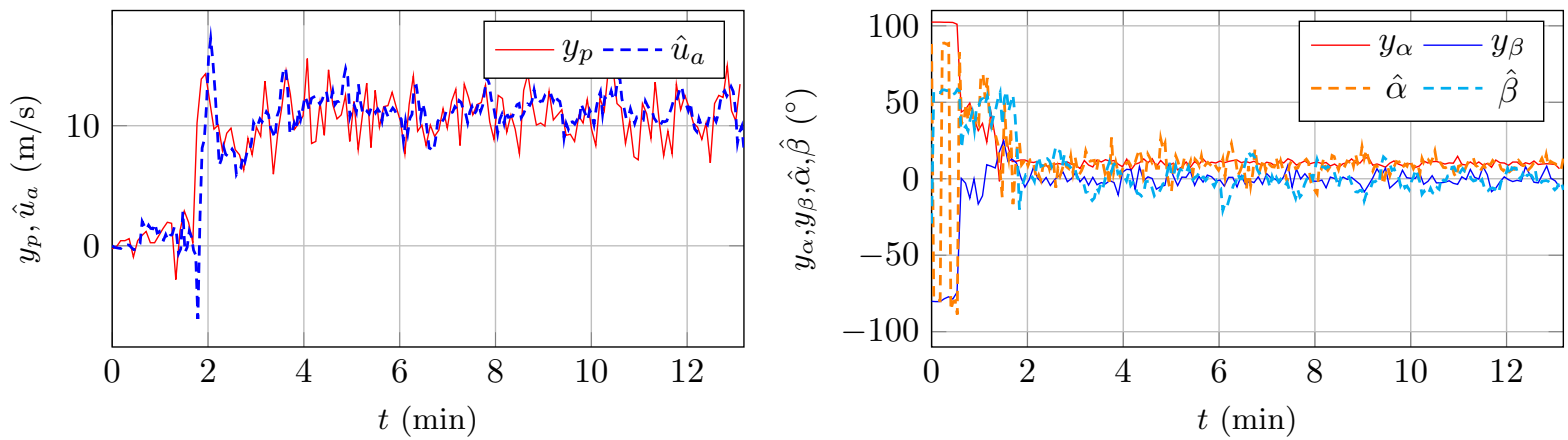

Figure 12. At left, 1D Pitot tube measurement $y_{p}$ and estimated longitudinal airspeed $\hat{u}_{a}$, as function of time. At right, estimated and measured Angle-of-Attack $\alpha$ and Angle-of-Sideslip $\beta$. The estimates are coherent with the measurements.

Another particular interesting aspect for the navigation is to compare the raw measurements about the airspeed with their estimated values, as depicted in Figure 12. We clearly observe similar global shapes between estimated and measured values, confirming the correct functioning of the filter.

\section{Conclusion \& Perspectives}

A novel tightly coupled estimator has been presented for the nonlinear state-space formulation of the local wind estimation and UAV navigation problem. Based on the Mulpliticative Kalman filtering, the proposed filter make use of the standard low-cost autopilot sensor suite (GPS, magnetometer, barometer, gyros and 
accelerometers) with additional 1D Pitot static tube, angle-of-attack and angle-of-sideslip sensors to estimate attitude, ground speed, position, sensors bias and wind. Particularly, the filter transforms the angular measurements, improving thus its accuracy and robustness, and simulations results illustrates the superiority of the proposed filter, which is also computationally efficient for future real onboard implementation. We compare then results from experimental flight data of the proposed filter with ground truth of a $60-\mathrm{m}$ measurement tower, which validate the performances of the proposed approach.

Future works concern the definition of an adaptive wind noise covariance depending on the UAV speed with specific attention to the numerical values and the direct inclusion of the proposed local wind estimation in a mapping framework.

\section{Acknowledgment}

The authors would like to acknowledge Gauthier Hattenberger and Xavier PARIs for their expertise about mini UAVs.

\section{A. Quaternions for Representing Rotations}

A unit quaternion provides a convenient mathematical notation for representing orientations and rotations of objects in three dimensions. ${ }^{36}$ Compared to Euler angles they are simpler to compose and avoid the problem of gimbal lock. Compared to rotation matrices, they are more compact, more numerically stable and more efficient. Indeed, for any unit quaternion

$$
\mathbf{q}=q_{r}+\mathbf{q}_{i}=\cos \left(\frac{\theta}{2}\right)+\mathbf{u} \sin (\theta),
$$

and for any vector $\mathbf{v} \in \mathbb{R}^{3}$ the action of the operator $\mathbf{q} *(). * \mathbf{q}^{-1}$ on $\mathbf{v}$ rotates the vector argument about axis $\mathbf{u}=\mathbf{q}_{i} /\left\|\mathbf{q}_{i}\right\|$ through an angle $\theta$, from which we can deduce a direction cosine matrix $\mathbf{R}_{\mathbf{q}}$ and Euler angles such that

$$
\mathbf{q} * \mathbf{v} * \mathbf{q}^{-1}=\mathbf{R}_{\mathbf{q}} \mathbf{v} .
$$

To let a quaternion, that is parameterized by a scalar and a three dimensional vector, operates on a vector $\mathbf{v} \in \mathbb{R}^{3}$, note that $\mathbf{v}$ can be viewed as a pure quaternion whose real part is zero, i.e., $\left[\begin{array}{l}0 \\ \mathbf{v}\end{array}\right]$. Thus, we can compactly define the following (non commutative) quaternion product $*$ as

$$
\mathbf{q}=\left[\begin{array}{c}
q_{r} \\
\mathbf{q}_{i}
\end{array}\right], \mathbf{p}=\left[\begin{array}{c}
p_{r} \\
\mathbf{p}_{i}
\end{array}\right], \mathbf{q} * \mathbf{p}=\left[\begin{array}{c}
q_{r} p_{r}-\mathbf{q}_{i}^{\top} \mathbf{p}_{i} \\
q_{r} \mathbf{p}_{i}+p_{r} \mathbf{q}_{i}+\left(\mathbf{p}_{i}\right)_{\times} \mathbf{q}_{i}
\end{array}\right],
$$

where the skew symmetric operator $(.)_{\times}$represents cross products as matrix multiplications.

\section{B. MEKF-Wind Error and Transition Matrix Definitions}

We develop here the machinery to obtain the state transition matrix $\mathbf{A}$, and the Jacobian of the state transition w.r.t. the process noise $\mathbf{A}_{\mathbf{n}}$, which are needed to the propagation step of MEKF-Wind, see Algorithm 1. According to the multiplicative error methodology ${ }^{20,21}$ we choose the following attitude error $\epsilon_{\mathbf{q}}$ defined as

$$
\mathbf{q}=\left[\begin{array}{c}
0 \\
\epsilon_{\mathbf{q}}
\end{array}\right] * \hat{\mathbf{q}}
$$

where (.) symbolizes estimated variables and which is after a small angular approximation equivalent to

$$
\mathbf{R}_{\mathbf{q}}=\left(\mathbf{I}+\left(\boldsymbol{\epsilon}_{\mathbf{q}}\right)_{\times}\right) \hat{\mathbf{R}}_{\mathbf{q}}
$$

and we then define the remaining errors as

$$
\epsilon_{\mathbf{v}}=\mathbf{v}-\hat{\mathbf{v}}, \epsilon_{\mathbf{x}}=\mathbf{x}-\hat{\mathbf{x}}, \epsilon_{\omega_{b}}=\omega_{b}-\hat{\boldsymbol{\omega}}_{b}, \epsilon_{\mathbf{a}_{b}}=\mathbf{a}_{b}-\hat{\mathbf{a}}_{b} \text { and } \epsilon_{\mathbf{w}}=\mathbf{w}-\hat{\mathbf{w}} .
$$


We thus derive the error dynamics using the nonlinear dynamics in (1)-(6), which are used to propagate then the error covariance matrix $\mathbf{P}$. The full error dynamic is given as

$$
\begin{aligned}
\dot{\boldsymbol{\epsilon}}_{\mathbf{q}} & =\mathbf{q} *\left(-\boldsymbol{\epsilon}_{\boldsymbol{\omega}_{b}}+\mathbf{n}_{\boldsymbol{\omega}}\right) * \mathbf{q}^{-1} \\
\dot{\boldsymbol{\epsilon}}_{\mathbf{v}} & =-\left(\mathbf{q} *\left(\mathbf{a}-\mathbf{a}_{b}\right) * \mathbf{q}^{-1}\right)_{\times} \boldsymbol{\epsilon}_{\mathbf{q}}+\mathbf{q} *\left(\boldsymbol{\epsilon}_{\mathbf{a}_{b}}-\mathbf{n}_{\mathbf{a}}\right) * \mathbf{q}^{-1} \\
\dot{\epsilon}_{\mathbf{x}} & =\boldsymbol{\epsilon}_{\mathbf{v}} \\
\dot{\epsilon}_{\boldsymbol{\omega}_{b}} & =-\boldsymbol{\epsilon}_{\boldsymbol{\omega}_{b}} / \tau_{\boldsymbol{\omega}}+\mathbf{n}_{\boldsymbol{\omega}_{b}}, \\
\dot{\epsilon}_{\mathbf{a}_{b}} & =-\boldsymbol{\epsilon}_{\mathbf{a}_{b}} / \tau_{\mathbf{a}}+\mathbf{n}_{\mathbf{a}_{b}}, \\
\dot{\epsilon}_{\mathbf{w}} & =\mathbf{n}_{\mathbf{w}}
\end{aligned}
$$

and we can derive from this model both the state transition matrix $\mathbf{A}$ and $\mathbf{A}_{\mathbf{n}}$, respectively, as

$$
\mathbf{A}=\left[\begin{array}{cccccc}
\mathbf{0} & \mathbf{0} & \mathbf{0} & \hat{\mathbf{R}}_{\mathbf{q}} & \mathbf{0} & \mathbf{0} \\
-\left(\hat{\mathbf{R}}_{\mathbf{q}}\left(\mathbf{a}-\hat{\mathbf{a}}_{b}\right)\right)_{\times} & \mathbf{0} & \vdots & \mathbf{0} & -\hat{\mathbf{R}}_{\mathbf{q}} & \vdots \\
\mathbf{0} & \mathbf{I} & \vdots & \mathbf{0} & \mathbf{0} & \vdots \\
\vdots & \mathbf{0} & \vdots & -\mathbf{I} / \tau_{\boldsymbol{\omega}} & \mathbf{0} & \vdots \\
\vdots & \vdots & \vdots & \mathbf{0} & -\mathbf{I} / \tau_{\mathbf{a}} & \vdots \\
\mathbf{0} & \mathbf{0} & \mathbf{0} & \mathbf{0} & \mathbf{0} & \mathbf{0}
\end{array}\right] \text { and }
$$

$$
\mathbf{A}_{\mathbf{n}}=\left[\begin{array}{ccccc}
\hat{\mathbf{R}}_{\mathbf{q}} & \mathbf{0} & \mathbf{0} & \mathbf{0} & \mathbf{0} \\
\mathbf{0} & \hat{\mathbf{R}}_{\mathbf{q}} & \vdots & \vdots & \vdots \\
\vdots & \mathbf{0} & \vdots & \vdots & \vdots \\
\vdots & \vdots & \mathbf{0} & \vdots & \vdots \\
\vdots & \vdots & \mathbf{I} & \mathbf{0} & \vdots \\
\vdots & \vdots & \mathbf{0} & \mathbf{I} & \mathbf{0} \\
\mathbf{0} & \mathbf{0} & \mathbf{0} & \mathbf{0} & \mathbf{I}
\end{array}\right]
$$

We thus are able to obtain the continuous dynamic of the covariance matrix $\mathbf{P}$ as

$$
\dot{\mathbf{P}}=\mathbf{A P A}^{\top}+\mathbf{A}_{\mathbf{n}} \mathbf{Q} \mathbf{A}_{\mathbf{n}}^{\top},
$$

that in discretized in Algorithm 1 and where $\mathbf{Q}$ is the covariance matrix of the process noise (7).

\section{Expectations \& Jacobians of the Transformed Measurements}

We develop here the calculus giving the expectations and Jacobians of the transformed measurements

$$
\begin{aligned}
\tilde{y}_{\alpha} & =\mathbf{C} \mathbf{v}_{a} \\
& =\mathbf{C}\left(\mathbf{q}^{-1} *(\mathbf{v}-\mathbf{w}) * \mathbf{q}\right) \text { and } \\
\tilde{y}_{\beta} & =\mathbf{v}_{a}^{\top} \mathbf{B} \mathbf{v}_{a} \\
& =\left(\mathbf{q}^{-1} *(\mathbf{v}-\mathbf{w}) * \mathbf{q}\right)^{\top} \mathbf{B}\left(\mathbf{q}^{-1} *(\mathbf{v}-\mathbf{w}) * \mathbf{q}\right),
\end{aligned}
$$

with $\mathbf{C}$ and $\mathbf{B}$ defined, respectively, in (25) and (26). Specifically, when the noise encoded in $y_{\alpha}$ and $y_{\beta}$ is present, these transformed measurements can be biased and we should theoretically evaluate the expected bias $\mathcal{E}\left\{y_{\alpha}\right\}$ and $\mathcal{E}\left\{y_{\beta}\right\}$ to remove it, that gives us the full alternative measurement (29). Consider firstly the measurement $\tilde{y}_{\alpha}$, we start from the Kalman filter assumptions, i.e., the state and measurement noise $n_{\alpha} \sim \mathcal{N}\left(0, \sigma_{\alpha}\right)$ follow Gaussian independent distributions. Let us then derive the following formula of scalar 
expectations

$$
\begin{aligned}
\mathcal{E}\{\cos (z)\} & =\mathrm{e}^{-\frac{\sigma^{2}}{2}} \cos (\hat{z}), \\
\mathcal{E}\{\sin (z)\} & =\mathrm{e}^{-\frac{\sigma^{2}}{2}} \sin (\hat{z}), \\
\mathcal{E}\left\{\cos ^{2}(z)\right\} & =\frac{1}{2}\left(1+\mathrm{e}^{-\sigma^{2}} \cos (2 \hat{z})\right), \\
\mathcal{E}\left\{\sin ^{2}(z)\right\} & =\frac{1}{2}\left(1-\mathrm{e}^{-\sigma^{2}} \cos (2 \hat{z})\right),
\end{aligned}
$$

for $z \sim \mathcal{N}(\hat{z}, \sigma)$, that we use in the sequel. For the expectation, since state error and noise are independent, we separate the expectation in noise expectation and state error expectation as

$$
\begin{aligned}
\mathcal{E}\left\{\tilde{y}_{\alpha}\right\} & =\mathcal{E}_{\mathbf{z}}\left\{\mathcal{E}_{\mathbf{n}}\{\mathbf{C}\} \mathbf{q}^{-1} *(\mathbf{v}-\mathbf{w}) * \mathbf{q}\right\}, \\
& =\mathcal{E}_{\mathbf{n}}\{\mathbf{C}\} \mathcal{E}_{\mathbf{z}}\left\{\mathbf{q}^{-1} *(\mathbf{v}-\mathbf{w}) * \mathbf{q}\right\},
\end{aligned}
$$

with after using (52)-(53)

$$
\begin{aligned}
\mathcal{E}_{\mathbf{n}}\{\mathbf{C}\} & =\mathrm{e}^{-\frac{\sigma_{\alpha}^{2}}{2}}[\sin (\hat{\alpha}) \quad 0 \quad-\cos (\alpha)], \\
& =\mathrm{e}^{-\frac{\sigma_{\alpha}^{2}}{2}} \hat{\mathbf{C}} \\
\mathcal{E}_{\mathbf{z}}\left\{\mathbf{q}^{-1} *(\mathbf{v}-\mathbf{w}) * \mathbf{q}\right\} & =\mathcal{E}_{\mathbf{z}}\left\{\left(\mathbf{I}-\left(\boldsymbol{\epsilon}_{\mathbf{q}}\right)_{\times}\right)\left(\hat{\mathbf{q}}^{-1} *\left(\hat{\mathbf{v}}-\hat{\mathbf{w}}+\boldsymbol{\epsilon}_{\mathbf{v}}-\boldsymbol{\epsilon}_{\mathbf{w}}\right) * \hat{\mathbf{q}}\right)\right\}, \\
& =\hat{\mathbf{q}}^{-1} *(\hat{\mathbf{v}}-\hat{\mathbf{w}}) * \hat{\mathbf{q}}-\mathcal{E}_{\mathbf{z}}\left\{\left(\boldsymbol{\epsilon}_{\mathbf{q}}\right)_{\times}\left(\hat{\mathbf{q}}^{-1} *\left(\boldsymbol{\epsilon}_{\mathbf{v}}-\boldsymbol{\epsilon}_{\mathbf{w}}\right) * \hat{\mathbf{q}}\right)\right\}, \\
& =\hat{\mathbf{v}}_{a}-\tilde{\mathbf{p}}, \text { with } \\
\tilde{\mathbf{p}} & =\hat{\mathbf{q}}^{-1} *\left(\mathbf{P}_{\mathbf{q v}}-\mathbf{P}_{\mathbf{q w}}\right)_{-\times} * \hat{\mathbf{q}},
\end{aligned}
$$

and where we remove the first order term in (58) since random variables have zero mean, use then the cross-covariances $\mathbf{P}_{\mathrm{qv}}$ and $\mathbf{P}_{\mathrm{qw}}$, between $\boldsymbol{\epsilon}_{\mathrm{q}}$ and, respectively, $\boldsymbol{\epsilon}_{\mathrm{v}}$ and $\boldsymbol{\epsilon}_{\mathrm{w}}$, and the counterpart of the cross product for matrix $(.)_{-\times}$, defined as

$$
\mathbf{P}=\left[\begin{array}{lll}
P_{11} & P_{12} & P_{13} \\
P_{21} & P_{22} & P_{23} \\
P_{31} & P_{32} & P_{33}
\end{array}\right],(\mathbf{P})_{-\times}=\left[\begin{array}{c}
P_{32}-P_{23} \\
P_{31}-P_{13} \\
P_{12}-P_{21}
\end{array}\right] .
$$

As a consequence, the expectation is the noise-free measurement multiplied by the factor $\mathrm{e}^{-\frac{\sigma_{\alpha}^{2}}{2}}$ plus an additional term, i.e., the expectation is given as

$$
\begin{aligned}
\mathcal{E}\left\{\tilde{y}_{\alpha}\right\} & =\mathrm{e}^{-\frac{\sigma_{\alpha}^{2}}{2}} \hat{\mathbf{C}}\left(\hat{\mathbf{v}}_{a}+\tilde{\mathbf{p}}\right), \\
& =\mathrm{e}^{-\frac{\sigma_{\alpha}^{2}}{2}}(0+\hat{\mathbf{C}} \tilde{\mathbf{p}}), \\
& =\mathrm{e}^{-\frac{\sigma_{\alpha}^{2}}{2}} \hat{\mathbf{C}} \tilde{\mathbf{p}} .
\end{aligned}
$$

We compute then the Jacobians w.r.t. the state and the noise, respectively, as

$$
\begin{aligned}
\frac{\partial \tilde{y}_{\alpha}}{\partial \mathbf{z}} & =\left[\begin{array}{llllll}
\mathbf{C} \hat{\mathbf{R}}_{\mathbf{q}}^{\top}(\hat{\mathbf{v}}-\hat{\mathbf{w}})_{\times} & \mathbf{C} \hat{\mathbf{R}}_{\mathbf{q}}^{\top} & \mathbf{0} & \cdots & 0 & -\mathbf{C R}_{\mathbf{q}}^{\top}
\end{array}\right], \\
\frac{\partial \tilde{y}_{\alpha}}{\partial n_{\alpha}} & =\left[\begin{array}{lllll}
\mathrm{e}^{-\frac{\sigma_{\alpha}^{2}}{2}} \sin \left(y_{\alpha}\right) & 0 & -\mathrm{e}^{-\frac{\sigma_{\alpha}^{2}}{2}} \cos \left(y_{\alpha}\right)
\end{array}\right] \hat{\mathbf{v}}_{a} \text { and } \\
\frac{\partial \tilde{y}_{\alpha}}{\partial \mathbf{n}_{\mathbf{y}}} & =\left[\begin{array}{lllll}
\mathbf{0} & \cdots & \mathbf{0} & \frac{\partial \tilde{y}_{\alpha}}{\partial n_{\alpha}} & 0
\end{array}\right] .
\end{aligned}
$$

Similarly for $\tilde{y}_{\beta}$ but more tricky, we start from the Kalman filter assumptions, with $n_{\beta} \sim \mathcal{N}\left(0, \sigma_{\beta}\right)$. For the expectation, since state and noise are uncorrelated, we have

$$
\mathcal{E}\left\{\tilde{y}_{\beta}\right\}=\mathcal{E}_{\mathbf{z}}\left\{\left(\mathbf{q}^{-1} *(\mathbf{v}-\mathbf{w}) * \mathbf{q}\right)^{\top} \mathcal{E}_{\mathbf{n}}\{\mathbf{B}\}\left(\mathbf{q}^{-1} *(\mathbf{v}-\mathbf{w}) * \mathbf{q}\right)\right\} .
$$


Before developing this expectation with the error variables, we define

$$
\begin{aligned}
\tilde{\mathbf{v}} & =\hat{\mathbf{v}}-\hat{\mathbf{w}}, \\
\boldsymbol{\epsilon}_{\tilde{\mathbf{v}}} & =\boldsymbol{\epsilon}_{\mathbf{v}}-\boldsymbol{\epsilon}_{\mathbf{w}} \text { and } \\
\mathbf{D} & =\hat{\mathbf{R}} \mathcal{E}_{\mathbf{n}}\{\mathbf{B}\} \hat{\mathbf{R}}^{\top},
\end{aligned}
$$

leading the expectation about noise after using of (54) and (55) as

$$
\mathcal{E}_{\mathbf{n}_{\beta}}\{\mathbf{B}\}=\left[\begin{array}{ccc}
1-\mathrm{e}^{-\sigma_{\beta}^{2}} \cos \left(2 y_{\beta}\right) & 0 & 0 \\
0 & -1-\mathrm{e}^{-\sigma_{\beta}^{2}} \cos \left(2 y_{\beta}\right) & 0 \\
0 & 0 & 0
\end{array}\right] .
$$

We can then develop

$$
\begin{aligned}
\mathcal{E}\left\{\tilde{y}_{\beta}\right\} & =\mathcal{E}_{\mathbf{z}}\left\{\left(\tilde{\mathbf{v}}+\boldsymbol{\epsilon}_{\tilde{\mathbf{v}}}\right)^{\top}\left(\mathbf{I}+\left(\boldsymbol{\epsilon}_{\mathbf{q}}\right)_{\times}\right) \mathbf{D}\left(\mathbf{I}-\left(\boldsymbol{\epsilon}_{\mathbf{q}}\right)_{\times}\right)\left(\tilde{\mathbf{v}}+\boldsymbol{\epsilon}_{\tilde{\mathbf{v}}}\right)\right\}, \\
& =\mathcal{E}_{\mathbf{z}}\left\{\left(\tilde{\mathbf{v}}^{\top}-\tilde{\mathbf{v}}^{\top}\left(\boldsymbol{\epsilon}_{\mathbf{q}}\right)_{\times}+\boldsymbol{\epsilon}_{\tilde{\mathbf{v}}}^{\top}-\boldsymbol{\epsilon}_{\tilde{\mathbf{v}}}^{\top}\left(\boldsymbol{\epsilon}_{\mathbf{q}}\right)_{\times}\right) \mathbf{D}\left(\tilde{\mathbf{v}}+\left(\boldsymbol{\epsilon}_{\mathbf{q}}\right)_{\times} \tilde{\mathbf{v}}+\boldsymbol{\epsilon}_{\tilde{\mathbf{v}}}+\left(\boldsymbol{\epsilon}_{\mathbf{q}}\right)_{\times} \boldsymbol{\epsilon}_{\tilde{\mathbf{v}}}\right)\right\},
\end{aligned}
$$

and preserve only zero, second and fourth order terms since random variables have zero mean, leading to

$$
\begin{aligned}
\mathcal{E}\left\{\tilde{y}_{\beta}\right\} & =\mathcal{E}_{\mathbf{z}}\left\{\tilde{\mathbf{v}}^{\top} \mathbf{D} \tilde{\mathbf{v}}+\tilde{\mathbf{v}}^{\top} \mathbf{D}\left(\boldsymbol{\epsilon}_{\mathbf{q}}\right)_{\times} \boldsymbol{\epsilon}_{\tilde{\mathbf{v}}}-\tilde{\mathbf{v}}^{\top}\left(\boldsymbol{\epsilon}_{\mathbf{q}}\right)_{\times} \mathbf{D}\left(\boldsymbol{\epsilon}_{\mathbf{q}}\right)_{\times} \tilde{\mathbf{v}}-\tilde{\mathbf{v}}^{\top}\left(\boldsymbol{\epsilon}_{\mathbf{q}}\right)_{\times} \mathbf{D} \boldsymbol{\epsilon}_{\tilde{\mathbf{v}}}\right. \\
& \left.+\boldsymbol{\epsilon}_{\tilde{\mathbf{v}}}^{\top} \mathbf{D}\left(\boldsymbol{\epsilon}_{\mathbf{q}}\right)_{\times} \tilde{\mathbf{v}}-\boldsymbol{\epsilon}_{\tilde{\mathbf{v}}}^{\top}\left(\boldsymbol{\epsilon}_{\mathbf{q}}\right)_{\times} \mathbf{D} \tilde{\mathbf{v}}-\boldsymbol{\epsilon}_{\tilde{\mathbf{v}}}^{\top}\left(\boldsymbol{\epsilon}_{\mathbf{q}}\right)_{\times} \mathbf{D}\left(\boldsymbol{\epsilon}_{\mathbf{q}}\right)_{\times} \boldsymbol{\epsilon}_{\tilde{\mathbf{v}}}\right\} .
\end{aligned}
$$

We thus recognize the free-noise measurement

$$
\tilde{\mathbf{v}}^{\top} \mathbf{D} \tilde{\mathbf{v}}=\hat{\mathbf{v}}_{a}^{\top} \mathbf{B} \hat{\mathbf{v}}_{a}=0
$$

and cancel some parts of (73) with their minus transpose, to obtain

$$
\begin{aligned}
\mathcal{E}\left\{\tilde{y}_{\beta}\right\} & =\mathcal{E}_{\mathbf{z}}\left\{-\tilde{\mathbf{v}}^{\top}\left(\boldsymbol{\epsilon}_{\mathbf{q}}\right)_{\times} \mathbf{D}\left(\boldsymbol{\epsilon}_{\mathbf{q}}\right)_{\times} \tilde{\mathbf{v}}-\boldsymbol{\epsilon}_{\tilde{\mathbf{v}}}^{\top}\left(\boldsymbol{\epsilon}_{\mathbf{q}}\right)_{\times} \mathbf{D}\left(\boldsymbol{\epsilon}_{\mathbf{q}}\right)_{\times} \boldsymbol{\epsilon}_{\tilde{\mathbf{v}}}\right\}, \\
& =\mathcal{E}_{\mathbf{z}}\left\{-\boldsymbol{\epsilon}_{\mathbf{q}}^{\top}(\tilde{\mathbf{v}})_{\times} \mathbf{D}(\tilde{\mathbf{v}})_{\times} \boldsymbol{\epsilon}_{\mathbf{q}}-\boldsymbol{\epsilon}_{\tilde{\mathbf{v}}}^{\top}\left(\boldsymbol{\epsilon}_{\mathbf{q}}\right)_{\times} \mathbf{D}\left(\boldsymbol{\epsilon}_{\mathbf{q}}\right)_{\times} \boldsymbol{\epsilon}_{\tilde{\mathbf{v}}}\right\}, \\
& =-\operatorname{Tr}\left((\tilde{\mathbf{v}})_{\times} \mathbf{D}(\tilde{\mathbf{v}})_{\times} \mathbf{P}_{\mathbf{q q}}\right)-\mathcal{E}_{\mathbf{z}}\left\{\boldsymbol{\epsilon}_{\tilde{\mathbf{v}}}^{\top}\left(\epsilon_{\mathbf{q}}\right)_{\times} \mathbf{D}\left(\boldsymbol{\epsilon}_{\mathbf{q}}\right)_{\times} \epsilon_{\tilde{\mathbf{v}}}\right\}, \\
& \simeq-\operatorname{Tr}\left((\tilde{\mathbf{v}})_{\times} \mathbf{D}(\tilde{\mathbf{v}})_{\times} \mathbf{P}_{\mathbf{q q}}\right)-\tilde{\mathbf{p}}^{\top} \mathbf{D} \tilde{\mathbf{p}},
\end{aligned}
$$

with $\operatorname{Tr}($.$) the trace operator and by considering the variance of the random variable \left(\boldsymbol{\epsilon}_{\mathbf{q}}\right)_{\times} \epsilon_{\tilde{\mathbf{v}}}$ as null (fourth order approximation).

Finally, let us compute the Jacobians w.r.t. the state and the noise, respectively, as

$$
\begin{aligned}
\frac{\partial \tilde{y}_{\beta}}{\partial \mathbf{z}} & =\left[\begin{array}{lrrrrr}
2 \hat{\mathbf{v}}_{a}^{\top} \mathbf{B} \hat{\mathbf{R}}_{\mathbf{q}}^{\top}(\hat{\mathbf{v}}-\hat{\mathbf{w}})_{\times} & 2 \hat{\mathbf{v}}_{a}^{\top} \mathbf{B} \hat{\mathbf{R}}_{\mathbf{q}}^{\top} & \mathbf{0} & \cdots & 0 & -2 \hat{\mathbf{v}}_{a}^{\top} \mathbf{B} \hat{\mathbf{R}}_{\mathbf{q}}^{\top}
\end{array}\right], \\
\frac{\partial \tilde{y}_{\beta}}{\partial n_{\beta}} & =\left[\begin{array}{llll}
-\sin \left(2 y_{\beta}\right) & 0 & \sin \left(2 y_{\beta}\right)
\end{array}\right] \operatorname{diag}\left(\hat{\mathbf{v}}_{a}\right) \hat{\mathbf{v}}_{a} \text { and } \\
\frac{\partial \tilde{y}_{\beta}}{\partial \mathbf{n}_{\mathbf{y}}} & =\left[\begin{array}{llll}
\mathbf{0} & \cdots & 0 & \frac{\partial \tilde{y}_{\beta}}{\partial n_{\beta}}
\end{array}\right] .
\end{aligned}
$$

\section{References}

\footnotetext{
${ }^{1}$ Al-Sabban, W., Gonzalez, L., and Smith, R., "Wind-Energy Based Path Planning for Unmanned Aerial Vehicles Using Markov Decision Processes," International Conference on Robotics and Automation (ICRA), IEEE, 2013, pp. 784-789.

${ }^{2}$ Ragi, S. and Chong, E., "Dynamic UAV Path Planning for Multitarget Tracking," American Control Conference (ACC), IEEE, 2012, pp. 3845-3850.

${ }^{3}$ Rysdyk, R., "Unmanned Aerial Vehicle Path Following for Target Observation in Wind," Journal of Guidance, Control, and Dynamics, Vol. 29, No. 5, 2006, pp. 1092-1100.
} 
${ }^{4}$ Ware, J. and Roy, N., "An Analysis of Wind Field Rstimation and Exploitation for Quadrotor Flight in the Urban Canopy Layer," International Conference on Robotics and Automation (ICRA), IEEE, 2016, pp. 1507-1514.

${ }^{5}$ Lawrance, N., Acevedo, J., Chung, J., Nguyen, J., Wilson, D., and Sukkarieh, S., "Long Endurance Autonomous Flight for Unmanned Aerial Vehicles," AerospaceLab, , No. 8, 2014, pp. p. 1-15.

${ }^{6}$ Reymann, C., Renzaglia, A., Lamraoui, F., Bronz, M., and Lacroix, S., "Adaptive Sampling of Cumulus Clouds with UAVs," Autonomous Robots, 2017, pp. 1-22.

${ }^{7}$ Elston, J., Argrow, B., Stachura, M., Weibel, D., Lawrence, D., and Pope, D., "Overview of Small Fixed-Wing Unmanned Aircraft for Meteorological Sampling," Journal of Atmospheric and Oceanic Technology, Vol. 32, No. 1, 2015 , pp. 97-115.

${ }^{8}$ Mayer, S., Hattenberger, G., Brisset, P., Jonassen, M., and Reuder, J., "A 'No-Flow-Sensor' Wind Estimation Algorithm for Unmanned Aerial Systems," International Journal of Micro Air Vehicles, Vol. 4, No. 1, 2012, pp. 15-29.

${ }^{9}$ Neumann, P., Asadi, S., Lilienthal, A., Bartholmai, M., and Schiller, J., "Autonomous Gas-Sensitive Microdrone: Wind Vector Estimation and Gas Distribution Mapping," Robotics \& Automation Magazine, Vol. 19, No. 1, 2012 , pp. 50-61.

${ }^{10}$ Lee, J. H., Sevil, H. E., Dogan, A., and Hullender, D., "Estimation of Maneuvering Aircraft States and Time-Varying Wind with Turbulence," Aerospace Science and Technology, Vol. 31, 2013, pp. 87 - 98.

${ }^{11}$ Beard, R. and McLain, T., Small Unmanned Aircraft: Theory and Practice, Princeton University Press, Princeton, NJ, USA, 2012

${ }^{12}$ Klein, V. and Morelli, E. A., Aircraft System Identification: Theory and Practice, American Institute of Aeronautics and Astronautics, Reston ,VA, 2006.

${ }^{13}$ Langelaan, J., Alley, N., and Neidhoefer, J., "Wind Field Estimation for Small Unmanned Aerial Vehicles," Journal of Guidance, Control, and Dynamics, Vol. 34, No. 4, 2011, pp. 1016-1030.

${ }^{14}$ van den Kroonenberg, A., Martin, T., Buschmann, M., Bange, J., and Vörsmann, P., "Measuring the Wind Vector Using the Autonomous Mini Aerial Vehicle MEAV," Journal of Atmospheric and Oceanic Technology, Vol. 25, No. 11, 2008, pp. 1969-1982.

${ }^{15}$ Rhudy, M. B., Gu, Y., Gross, J. N., and Chao, H., "Onboard Wind Velocity Estimation Comparison for Unmanned Aircraft Systems," IEEE Transactions on Aerospace and Electronic Systems, Vol. 53, No. 1, 2017, pp. 55-66.

${ }^{16}$ Cho, A., Kim, J., Lee, S., and Kee, C., "Wind Estimation and Airspeed Calibration Using a UAV with a Single-Antenna GPS Receiver and Pitot Tube," IEEE Transactions on Aerospace and Electronic Systems, Vol. 47, No. 1, 2011, pp. $109-117$.

${ }^{17}$ Rhudy, M., Larrabee, T., Chao, H., Gu, Y., and Napolitano, M., "UAV Attitude, Heading, and Wind Estimation Using GPS/INS and an Air Data System," American Institute of Aeronautics and Astronautics, 2013.

${ }^{18}$ Condomines, J.-P., Bronz, M., Hattenberger, G., and Erdelyi, J.-F., "Experimental Wind Field Estimation and Aircraft Identification," International Micro Air Vehicles Conference and Flight Competition (IMAV), 2015.

${ }^{19}$ Johansen, T. A., Cristofaro, A., Srensen, K., Hansen, J. M., and Fossen, T. I., "On Estimation of Wind Velocity, Angleof-Attack and Sideslip Angle of Small UAVs Using Standard Sensors," International Conference on Unmanned Aircraft Systems (ICUAS), 2015, pp. 510-519.

${ }^{20}$ Martin, P. and Salaün, E., "Generalized Multiplicative Extended Kalman Filter for Aided Attitude and Heading Reference System," AIAA Guidance, Navigation, and Control Conference, 2010.

${ }^{21}$ Trawny, N. and Roumeliotis, S., "Indirect Kalman Filter for 3D Attitude Estimation," University of Minnesota, Dept. of Comp. Sci. \& Eng., Tech. Rep, Vol. 2, 2005.

22 "Invariant Extended Kalman Filter: Theory and Application to a Velocity-Aided Attitude Estimation Problem," Conference On Decision and Control.

${ }^{23}$ Markley, F. L., "Attitude Error Representations for Kalman Filtering," Journal of Guidance, Control, and Dynamics, Vol. 26, No. 2, 2003, pp. 311-317.

${ }^{24}$ Crassidis, J. L. and Markley, F. L., "Unscented filtering for spacecraft attitude estimation," Journal of guidance, control, and dynamics, Vol. 26, No. 4, 2003, pp. 536-542.

${ }^{25}$ Guillaume, D. and Rouchon, P., "Observable Systems Transformable into Implicit Affine Forms," IFAC Proceedings Volumes, Vol. 31, No. 17, 1998, pp. 775 - 779.

${ }^{26}$ Tan, F., Lohmiller, W., and Slotine, J.-J., "Analytical SLAM Without Linearization," arXiv preprint:1512.08829, 2015.

${ }^{27}$ El-Sheimy, N., Hou, H., and Niu, X., "Analysis and Modeling of Inertial Sensors Using Allan Variance," IEEE Transactions on Instrumentation and Measurement, Vol. 57, No. 1, 2008, pp. 140-149.

${ }^{28}$ Allan, D., Shoaf, J., and Halford, D., "Statistics of Time and Frequency Data Analysis," Time and Frequency: Theory and Fundamentals, 1974.

${ }^{29}$ Tian, P., Chao, H., Gu, Y., and Hagerott, S. G., "UAV Flight Test Evaluation of Fusion Algorithms for Estimation of Angle of Attack and Sideslip Angle," AIAA Guidance, Navigation, and Control Conference, 2016.

${ }^{30}$ Longbin, M., Xiaoquan, S., Yiyu, Z., Kang, S. Z., and Bar-Shalom, Y., "Unbiased Converted Measurements for Tracking," IEEE Transactions on Aerospace and Electronic Systems, Vol. 34, No. 3, 1998, pp. 1023-1027.

${ }^{31}$ Yang, C., Shi, W., and Chen, W., "Comparison of Unscented and Extended Kalman Filters with Application in Vehicle Navigation," Journal of Navigation, Vol. 70, No. 02, 2017, pp. 411-431.

${ }^{32}$ Sosa, C. R., "Quaternion Kinematics for the Error-State KF," 2015.

${ }^{33}$ Tascikaraoglu, A. and Uzunoglu, M., "A review of Combined Approaches for Prediction of Short-Term Wind Speed and Power," Renewable and Sustainable Energy Reviews, Vol. 34, 2014, pp. 243 - 254.

${ }^{34}$ Bianchi, F. D., Mantz, R. J., and De Battista, H., "The Wind and Wind Turbines," Wind Turbine Control Systems. Principles, Modelling and Gain Scheduling Design, Springer, 2007.

${ }^{35}$ Brisset, P., Drouin, A., Gorraz, M., Huard, P.-S., and Tyler, J., "The Paparazzi Solution," MAV 2006, 2nd US-European Competition and Workshop on Micro Air Vehicles, Sandestin, United States, 2006.

${ }^{36}$ Kuipers, J. B., Quaternions and Rotation Sequences : A Primer with Applications to Orbits, Aerospace, and Virtual Reality, Princeton Univ. Press, 1999. 